\title{
Very high energy gamma-rays from Centaurus X-3: Indications and implications
}

\author{
A. M. Atoyan ${ }^{1, \star}$, K.-M. Aye ${ }^{2}$, P. M. Chadwick ${ }^{2}$, M. K. Daniel ${ }^{2}$, K. Lyons ${ }^{2}$, T. J. L. McComb ${ }^{2}$, \\ J. M. McKenny ${ }^{2}$, S. J. Nolan ${ }^{2}$, K. J. Orford ${ }^{2}$, J. L. Osborne' ${ }^{2}$, and S. M. Rayner ${ }^{2}$ \\ 1 McGill University, Physics Department, Montreal H3A 2T8, Canada \\ 2 Physics Department, Durham University, Durham DH1 3LE, UK
}

Received 3 August 2001 / Accepted 26 November 2001

\begin{abstract}
We present the results of a detailed timing analysis of observations of Cen X-3 taken by the University of Durham Mark 6 imaging atmospheric Cherenkov telescope in 1997-1999. The presence of a TeV $\gamma$-ray signal at the overall $\geq 4.5 \sigma$ significance level in the "fully cut" image selected data, as reported earlier, is confirmed. A search for possible modulations of $\gamma$-rays with the pulsar spin period $P_{0} \approx 4.8 \mathrm{~s}$ was performed by the step-by-step application of image parameter cuts of gradually increasing hardness. The data of each of 23 days of observations have not revealed any statistically significant Rayleigh power peak, except for 1 day when a peak with a chance probability $p=6.8 \times 10^{-7}$ was found in "soft-cut" data sets. This modulation, if real, is blue shifted by $6.6 \mathrm{~ms}$ $\left(>10^{3} \mathrm{~km} \mathrm{~s}^{-1}\right)$ from the nominal second harmonic of the X-ray pulsar. Taking the large number of frequency trials into account, the estimated final probability of finding such a peak by chance still remains $<10^{-2}$. Bayesian statistical analysis also indicates the presence of such modulations. We show that the behaviour of the Rayleigh peak disappearing in the fully cut data set is actually quite consistent with the hypothesis of a $\gamma$-ray origin of that peak. No modulation of the VHE $\gamma$-ray signal with the pulsar orbital phase is found.

In the second part of the paper we consider different theoretical models that could self-consistently explain the existing data from Cen X-3 in high-energy (HE, $E \geq 100 \mathrm{MeV}$ ) and very high energy (VHE, $E \geq 100 \mathrm{GeV}$ ) $\gamma$-rays. We propose on the basis of the energetics required that all reasonable options for the $\gamma$-ray production in Cen X-3 must be connected to jets emerging from the inner accretion disc around the neutron star. One of the principal options is a large-scale source, with $R_{\mathrm{s}} \sim 10^{13}-10^{14} \mathrm{~cm}$; this assumes effective acceleration of electrons up to $\sim 10 \mathrm{TeV}$ by shocks produced by interaction of these jets with the dense atmosphere of the binary. It is shown that such a quasi-stationary model could explain the bulk of the $\gamma$-radiation features observed except for the $\gamma$-ray modulations with the pulsar spin. These modulations, if genuine, would require an alternative source with $R_{\mathrm{s}} \ll 10^{11} \mathrm{~cm}$. We consider two principal models, hadronic and leptonic, for the formation of such a compact source in the jet. Both models predict that the episodes of pulsed $\gamma$-ray emission may be rather rare, with a typical duration not exceeding a few hours, and that generally the frequency of pulsations should be significantly shifted from the nominal frequency of the X-ray pulsar. The opportunities to distinguish between different models by means of future $\gamma$-ray observations of this X-ray binary are also discussed.
\end{abstract}

Key words. acceleration of particles - radiation mechanisms: non-thermal - stars: individual: Cen X-3 gamma-rays: theory - gamma-rays: observations - X-rays: binaries

\section{Introduction}

Centaurus X-3 has been one of the prominent galactic sources of hard radiation since its discovery as one of the first cosmic X-ray sources (Chodil et al. 1967), and was the first X-ray pulsar to be discovered in a binary system (Giacconi et al. 1971; Schreier et al. 1972). All the basic parameters of this archetypal high mass X-ray binary are

Send offprint requests to: M. K. Daniel or A. M. Atoyan, e-mail: m.k.daniel@dur.ac.uk;

atoyan@hep.physics.mcgill.ca

* On leave from Yerevan Physics Institute, Armenia. well known. The pulsar has a spin period $P_{0} \approx 4.8 \mathrm{~s}$ and an orbital period $P_{\text {orb }} \approx 2.1 \mathrm{~d}$, with a gradual shortening (i.e. "spinning-up") of both periods in time, and with a deep eclipse of the X-ray source at the orbital phases $-0.12 \leq \phi \leq 0.12$ (for reviews see Joss \& Rappaport 1984; Nagase 1989). The X-ray luminosity of the pulsar is very large, reaching $L_{\mathrm{X}} \sim 10^{38} \mathrm{erg} \mathrm{s}^{-1}$ in the "high" state (e.g. White et al. 1983; Burderi et al. 2000), which implies a massive accretion of material onto the neutron star from the optical companion (Pringle \& Rees 1972; Lamb et al. 1973). The optical companion (V779 Cen) 
was discovered by Krzeminski (1974) and has been identified as an evolved O-type star with surface temperature $T \geq 3 \times 10^{4} \mathrm{~K}$ at a distance $\sim 8 \mathrm{kpc}$ from the Sun, and a bolometric magnitude of $M_{\mathrm{bol}} \sim-9$ (Hutchings et al. 1979). The masses of the stars in this binary are estimated as $M_{\mathrm{n}} \simeq 1.2 M_{\odot}$ and $M_{\mathrm{O}-\text { star }} \simeq 20 M_{\odot}$. The value of $P_{\text {orb }}$ suggests the separation between the centres of the stars to be $a=1.3 \times 10^{12} \mathrm{~cm}$ and the radius of the massive star filling its Roche lobe $R_{\mathrm{O}}=8.6 \times 10^{11} \mathrm{~cm}$ (see e.g. Clark et al. 1988; Ash et al. 1999).

Cen X-3 is also known as one of those X-ray binaries from which $\gamma$-ray signals have been reported. In the domain of high energy (HE) $\gamma$-rays, conventionally $E \geq 100 \mathrm{MeV}$, the $\gamma$-ray flux during 2 weeks of observations of Cen X-3 in October 1994 by EGRET was at the level $F(>100 \mathrm{MeV})=(9.2 \pm 2.3) \times 10^{-7} \mathrm{ph} \mathrm{cm}^{-2} \mathrm{~s}^{-1}$ (Vestrand et al. 1997). This excess flux was significant at a $5 \sigma$ level, which is generally considered as a reliable detection with the EGRET instrument. The timing analysis has shown a significant modulation of the signal with the pulsar spin, precisely at the contemporaneous frequency of X-ray pulsations measured by BATSE, with a chance probability estimate based on the Rayleigh test statistic of about $1.6 \times 10^{-3}$. No modulation with the orbital phase of the pulsar was found: the $\gamma$-ray signal seems to be quite homogeneously distributed throughout the entire orbit, with 68 of the $264 \mathrm{HE} \gamma$-rays from Cen X-3 being detected in the pulsar eclipse orbital phase $|\phi| \leq 0.12$. This suggests a production site of the $\gamma$-rays far away from the pulsar. The $\gamma$-ray signal was not found in the data of other observing periods of Cen X-3 by EGRET, which suggests a significant variability of the $\mathrm{HE} \gamma$-ray source on a time scale of several months (see Vestrand et al. 1997 for details).

In the domain of very high energies (VHE), conventionally $E \geq 100 \mathrm{GeV}$, sporadic $\gamma$-ray signals from Cen X-3 had been earlier reported by the University of Durham (Brazier et al. 1990a) and the Potchefstroom groups (North et al. 1990). Note that the early detections of VHE $\gamma$-rays from X-ray binaries in the 80 's had been carried out by non-imaging Cherenkov telescopes which had rather poor sensitivity. The $\gamma$-ray signals were therefore extracted mostly on the basis of the timing analyses, and are subject to controversy concerning their reliability (see Weekes 1992). It is worth noting in this regard that Cen X-3 is the first and until now the only X-ray binary to be detected as a source of $E \geq 400 \mathrm{GeV} \gamma$-rays by a contemporary imaging instrument, the Durham Mark 6 telescope, as we have reported earlier (Chadwick et al. $1998,2000)$. The excess ( $\gamma$-ray) signal has been found in the "ON-source" data during each of the 3 years of observations from 1997 to 1999 . The estimated mean $\gamma$-ray flux is about $F(>400 \mathrm{GeV}) \simeq 2.8 \times 10^{-11} \mathrm{~cm}^{-2} \mathrm{~s}^{-1}$, at the significance level for the entire data set of $4.7 \sigma$. No significant modulations in the combined ON-source data with either the $4.8 \mathrm{~s}$ pulsar period or $2.1 \mathrm{~d}$ orbital period of the binary were found, but these data were analyzed after application of the image cut procedures (Chadwick et al. 2000) which we shall see later can be very counter productive in a periodicity search.

In this paper we present the results of a more detailed timing analysis of observations of Cen X-3, which in particular includes a step-by-step search for possible shortterm episodes of $\gamma$-ray emission modulated with the spin of the pulsar, as well as a search for a modulation of the $\gamma$-ray signal with the pulsar orbital phase (Sect. 2). Then we carry out in Sect. 3 a detailed theoretical study of the consistency of different models for the production of $\gamma$-rays in Cen X-3 with the experimental data currently available in both HE and VHE domains. Finally, in Sect. 4 we summarize the results of our current study, and discuss some possible tests for future $\gamma$-ray observations that could help to distinguish between different models of $\gamma$-ray production in this X-ray binary.

\section{Timing analysis}

As we have reported earlier (Chadwick et al. 1998, 2000), the entire set of data of observations of Cen X-3 by the University of Durham Mark 6 telescope (see Armstrong et al. 1999 for a description) from Narrabri, Australia, consist of pairs of $15 \mathrm{~min}$ segments of alternately ONand OFF-source observations. A total of 129 ON-source segments have passed the relevant consistency criteria in order to be accepted for further analysis, yielding about $32 \mathrm{hrs}$ worth of data. The data have been accumulated during 23 days of observations over 3 years from 1997 to 1999. After application of a set of shower image parameter cuts (see Chadwick et al. 2000), an excess of events in the on-source data, indicating a possible presence of $\gamma$-ray signal, is found in the data from each individual year. In Table 1 we present the results of the analysis of the data for all of the 23 days of observations. Note that the significance $4.34 \sigma$ in the overall data set for the image brightness parameter brightness $>800$ digital counts, which corresponds to the Monte-Carlo estimated $\gamma$-ray energies $E>400 \mathrm{GeV}$, is somewhat lower than $4.7 \sigma$ as reported previously (Chadwick et al. 2000) because two days worth of data were subsequently corrected for a problem with one of the imaging PMT's.

For the periodicity analysis the air shower arrival times were first corrected to the reference frame of the solar system barycentre in order to exclude the timing effects connected with the motion of the Earth.

\subsection{Orbital phase modulations}

Important information about a plausible site and mechanism of gamma-ray production in X-ray binaries can be derived from studies of possible correlations of the $\gamma$-ray fluxes with the orbital phase of the pulsar. This can be particularly informative in the case of Cen X-3 because it is an eclipsing binary, and because its optical companion is a very luminous star. As shown by Bednarek (2000), in the case of $\gamma$-ray production near the orbit of Cen X-3, the 

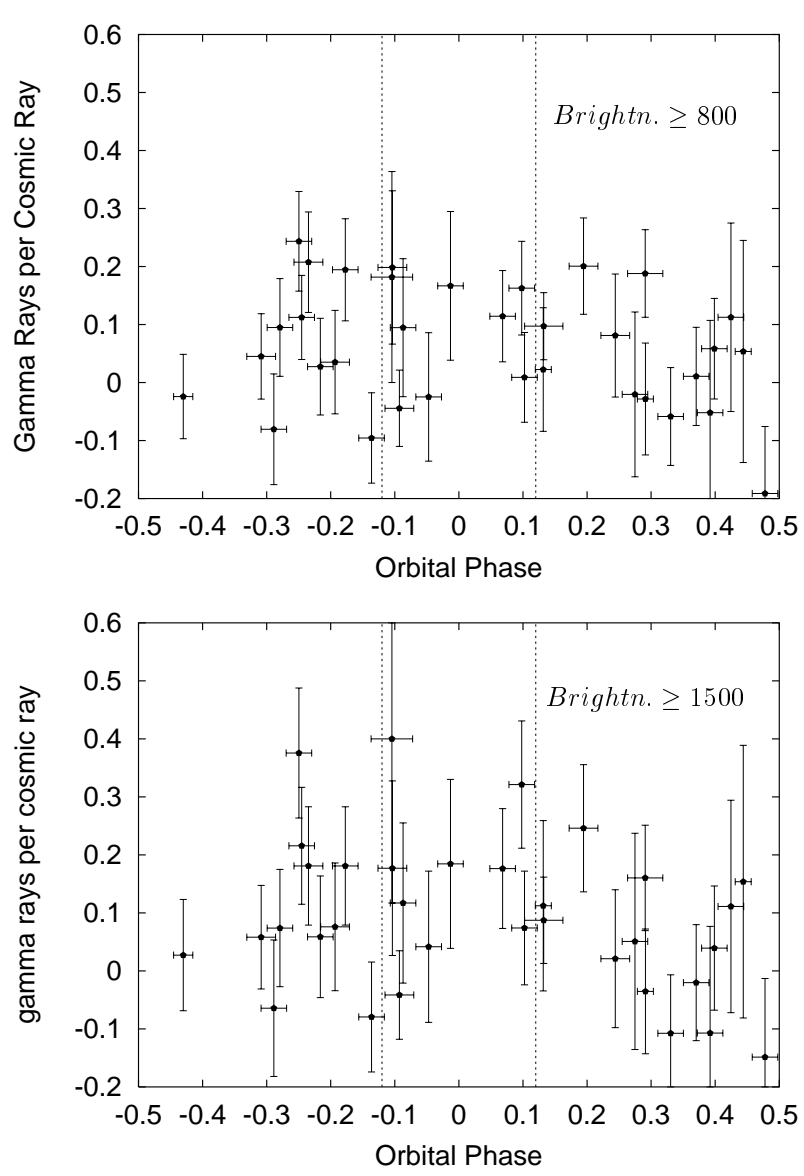

Fig. 1. ON-OFF excess, in terms of " $\gamma$-rays per cosmic ray", in the fully cut data sets with 2 different brightness parameters, plotted with respect to orbital phases of observations in 19971999 .

optical depth of the VHE $\gamma$-rays can vary from $\tau_{\gamma \gamma} \sim 10$ to $\tau_{\gamma \gamma}<1$ with the variation of the orbital phase from $\phi \geq 0.12$ (i.e. outside the pulsar eclipse) to $\phi \sim 0.5$ due to absorption on the optical/UV photons from the companion. If $\gamma$-radiation is produced close to the pulsar then a strong correlation of the signal strength with the orbital phase should be seen.

For calculations of the orbital phase we use the ephemeris by Nagase et al. (1992), which are in quite good agreement with the ones by Kelley et al. (1983) we used earlier (Chadwick et al. 1998, 2000), but being more contemporary are more accurate and appropriate for the period of our observations. Because the length of all ON/OFF segments accumulated during individual observation nights varies and may reach several hours, i.e. a significant fraction of the $2.1 \mathrm{~d}$ orbital period, we have split the data of the nights with a large $(\geq 5)$ overall number of on-source segments into sequences of 3-4 on-source segments (which in some cases may overlap). In principle, such a grouping of data allows one to also look for variations of the signal on timescales $\sim 1.5-2 \mathrm{~h}$, which could be of interest from a theoretical point of view (see Sect. 3 below).
A trend of a uniform distribution of the $\gamma$-ray signal with the orbital phase of the pulsar is apparent in Fig. 1 where we show the excess events in the on-source segments in terms of their ratio to the number of events in the counterpart off-source segments. This method (see Chadwick et al. 1999) allows an assessment of the strength of a possible $\gamma$-ray signal with respect to the intensity of cosmic rays independent of the daily variations in the performance of the telescope. Note that there is some deficit in the number of on-source segments corresponding to the orbital phase $-0.5 \leq \phi \leq-0.3$. For all other phases, including the eclipse phase, the segments with high (say $\geq 0.1$ ) ON-OFF excess are distributed quite homogeneously. It is worth noting also that most of these high excess data points have moved further up in significance when only the events with the parameter brightness $\geq 1500$ digital counts are selected. A reasonable explanation for this effect is that the $\gamma$-ray events are indeed present in those segments, and that the spectrum of these $\gamma$-rays is significantly harder than the spectrum of cosmic rays.

\subsection{Pulsar spin modulation}

In our previous analyses we were looking for a modulation of the VHE $\gamma$-ray signal with the X-ray pulsar in a very narrow band of frequencies around the fundamental frequency $\nu_{0}=1 / P_{0}$ using the entire ON-source dataset. Meanwhile the earlier reports on the $\gamma$-ray signals modulated with the X-ray pulsar spin in Cen X-3 (Brazier et al. 1990a), as well as in some other X-ray binaries such as Cygnus X-3 (Brazier et al. 1990b) or Hercules X-1 (e.g. Dowthwaite et al. 1984; Lamb et al. 1988), had indicated that the pulsed $\gamma$-ray emission was sporadic, with a duration typically not exceeding $1 \mathrm{~h}$ and occasionally significantly shorter. Moreover, the frequency of $\gamma$-pulsations reported in Her X-1 had on occasion been observed to be significantly shifted from the nominal frequency of the X-ray pulsar by up to $\simeq 0.16 \%$ (Lamb et al. 1988; Resvanis et al. 1988; Dingus et al. 1988).

Therefore it seemed worth conducting a search for any possible modulation of the $\gamma$-ray emission with the BATSE derived pulsar spin period in a wider bandpass around $P_{0} \approx 4.81 \mathrm{~s}$. The test initially used was the standard Rayleigh test for periodicity. The search was conducted at both the period and the half-period (in the interval from $4.79 \mathrm{~s}$ to $4.83 \mathrm{~s}$ and $2.395 \mathrm{~s}$ to $2.415 \mathrm{~s}$ respectively) due to the insensitivity of the Rayleigh test to light curves with a double peak separated by $\pi$ in phase; a trait occasionally observed in Cen X-3's X-ray light curve (Tuohy 1976; Nagase et al. 1992; Burderi et al. 2000). This feature suggests that both accretion hot spots of the pulsar may become visible during one cycle of its rotation, which is quite possible in the case of a relatively large inclination angle of the pulsar magnetic axis. It is not improbable that the VHE $\gamma$-rays might have the basic modulation near the half-period rather than the full spin period of the pulsar. 
The time range tested corresponds to Doppler shifted speeds of the putative $\gamma$-ray source relative to the neutron star of up to $v \sim 1200 \mathrm{~km} \mathrm{~s}^{-1}$. Note that the orbital speed of the Cen X-3 pulsar is $414 \mathrm{~km} \mathrm{~s}^{-1}$, and the speed of the wind driven by the optical companion star in this binary is $\sim 1000 \mathrm{~km} \mathrm{~s}^{-1}$ (Clark et al. 1988).

For a typical observation of Cen X-3 during one night the duration is $\Delta t_{\mathrm{obs}} \sim 3 \mathrm{hr}$ (in a succession of "ON" and "OFF" segments) therefore the chosen range of trial periods corresponds to $\simeq 18-19$ independent Fourier frequency (IFF) intervals $\Delta \nu=1 / \Delta t_{\mathrm{obs}}$ around $P_{0}$ (and about twice that for the half-period search). Given the wide band of trial periods studied, there is no need for an additional adjustment of the event arrival times to the pulsar position because any significant modulation frequency to be found could later on be easily compared with the Doppler shifted frequency of the X-ray pulsar at the known orbital position. Moreover, to adjust the event times to the pulsar site may well be unjustified since both the HE and VHE data give no indication of orbital modulation, with about a quarter of all excess photons from the EGRET data being in the eclipse phase of the pulsar and the episode of highest significance in the Mark 6 data occuring during the $\mathrm{X}$-ray eclipse. This implies the production site of the $\gamma$-radiation to be far from the pulsar itself.

We have first considered, using the standard Rayleigh test statistic, the data of each individual night of observation of Cen X-3 after application of the full set of the Extensive Air Shower image parameter cuts as described in Chadwick et al. (1998). This analysis did not reveal any Rayleigh power that could be potentially significant either around the main period $P_{0} \simeq 4.8 \mathrm{~s}$ or around the second harmonic $(P \sim 2.4 \mathrm{~s})$. This, however, is a result that one could reasonably expect.

The Rayleigh power at some frequency $\nu$ is defined as $P_{\mathrm{Rl}}=\left(c^{2}+s^{2}\right) / N$, where $c=\sum_{j=1}^{N} \cos \theta_{j}$ and $s=$ $\sum_{j=1}^{N} \sin \theta_{j}$, and $\theta_{j}=2 \pi \nu t_{j}$ is the phase of the $j$-th event. If the total number of events $N$ is large and the duration of the data set is $\gg$ the test period $1 / \nu$, then the sums $c$ and $s$ are practically independent (only weakly correlated), so for simplicity of further discussion we can take $P_{\mathrm{Rl}} \simeq 2 c^{2} / N$. For uniformly distributed events the mean $\bar{c}$ (and $\bar{s}$ ) is equal to 0 . However the root mean square (rms, the dispersion) of $c$ is not zero, but is equal to $d=\sqrt{\overline{c^{2}}}=\sqrt{N / 2}^{1}$, so the expected mean $\overline{P_{\mathrm{Rl}}}=1$. If now there are some $N_{\mathrm{G}}$ events (" $\gamma$-rays", G) modulated at frequency $\nu$, and the rest $N_{\mathrm{B}}=N-N_{\mathrm{G}}$ represents the uniform background (cosmic rays, B) and $N$ is the total number of events, then the expected mean Rayleigh power exceeds 1 . Taking into account that the mean values $\bar{c} \sim \bar{s} \sim \mathrm{G} / 2$ (for sinusoidal modulations) and that $\mathrm{G} \ll \mathrm{B} \simeq N$, one finds:

$$
\overline{P_{\mathrm{Rl}}} \simeq 2 \frac{\overline{c_{\mathrm{B}}^{2}}+\overline{\mathrm{c}_{\mathrm{B}} c_{\mathrm{G}}}+\overline{c_{\mathrm{G}}^{2}}}{N_{\mathrm{G}}+N_{\mathrm{B}}}=1+\sigma_{0}^{2},
$$

\footnotetext{
${ }^{1}$ It means that $c$ and $s$ are expected to be in the range $\pm d$.
}

where $\sigma_{0}=N_{\mathrm{G}} / \sqrt{2 N_{\mathrm{B}}}$. Note that, the latter variable represents the statistical significance of the signal in the data, which in practice is calculated as $\sigma=\frac{\left(N_{\text {on }}-N_{\text {off }}\right)}{\sqrt{N_{\text {on }}+N_{\text {off }}}}$, which implies $N_{\text {on }}=N_{\mathrm{B}}+N_{\mathrm{G}}$ and $N_{\text {off }}=N_{\mathrm{B}}$.

Any cuts made will affect the number of both $\gamma$-ray events, $N_{\mathrm{G}}^{\text {cut }}=F_{\mathrm{G}} N_{\mathrm{G}}$, and background cosmic ray events, $N_{\mathrm{B}}^{\text {cut }}=F_{\mathrm{B}} N_{\mathrm{B}}$, where $F_{\mathrm{G}}$ and $F_{\mathrm{B}}$ are the respective fractions of events surviving cuts. Since $F_{\mathrm{G}}$ can be $\gg F_{\mathrm{B}}$ imaging is a very useful tool for background rejection. The significance of a signal, however, is $\sigma \propto F_{\mathrm{B}}^{-1 / 2}$; therefore the cuts made need to be harsh to get the best signal to noise ratio, inevitably leading to a loss of $\gamma$-ray events from the dataset. In a d.c. search this is acceptable since merely increasing the observation time will lead to an increase in the number of $\gamma$-rays in a dataset; however, in a periodicity search - especially if the episodes of pulsed emission are short term and particularly when combined with a low flux of VHE $\gamma$-rays - any loss of $\gamma$-ray signal could prove fatal to a positive detection. Whilst cutting should improve the Rayleigh power mathematically, the low number of events surviving cuts means the dispersion of the Rayleigh power about the mean would give no reliable estimate of the true Rayleigh power, being typically within the range $P_{\mathrm{Rl}} \simeq P_{\mathrm{B}}+\sigma_{0}^{2} \pm 2 \sigma_{0} \sqrt{P_{\mathrm{B}}}$; where the sign of the last term depends on whether $c_{B}$ and $c_{G}$ are 'coherent' (have the same or different signs). In order to get a more robust statistic the cuts need to be relaxed, to allow for the statistical fluctuations of $N_{\mathrm{B}}$ and $N_{\mathrm{G}}$.

As an example, taking the average "on-source" observation time to be 1.5 hours, and using even the mean computed flux of 1997, $F(>400 \mathrm{GeV})=5 \times 10^{-11} \mathrm{~cm}^{-2} \mathrm{~s}^{-1}$, which is a factor of 2 higher than the mean during 19971999 from Cen X-3, the mean number of $\gamma$-rays for a detector with a collection area of $10^{9} \mathrm{~cm}^{2}$ and $50 \%$ efficiency (Chadwick et al. 2000) is $\sim 130$. Monte Carlo simulations indicate that the fraction of $\gamma$-rays passing full image parameter cuts is $\sim 20 \%$, so only $25-30$ of these could be pulsed $\gamma$-rays. Note that this is significantly less than minimum $40-50$ which is usually needed, given the impact of statistical fluctuations, for a successful practical application of the Rayleigh statistics, even if we neglect the background events. Meanwhile, if one takes into account also that there are on average about 400 events remaining in each night's data after the application of imaging cuts, the maximum expected possible Rayleigh power (assuming $c_{\mathrm{G}}^{2}+s_{\mathrm{G}}^{2} \simeq N_{\mathrm{G}}^{2}$ ) would only be ${\overline{P_{\mathrm{Rl}}}}^{\max } \simeq 1+(25)^{2} / 400 \sim 2.6$. This would correspond to a chance probability of only $\exp \left(-P_{\mathrm{Rl}}\right) \sim 0.07$ - before the number of trials has been taken into account. Compare this to the maximum Rayleigh power achieved by leaving $130 \gamma$-ray events in a background signal of $\sim 1000$ events, found by relaxing the image parameter cuts, of 17.9 that would give a chance probability of $\sim 10^{-8}$.

Thus, the hard image cut parameters which maximize the DC signal become destructive for the search of $\gamma$-ray modulations with the pulsar spin period if the data of individual observational nights are to be analyzed. For this 
Table 1. The numbers of events in the "ON" and "OFF" pairs of segments, the significance and the relative excess (in terms of "gamma-rays per cosmic ray") of the signal in the data of 23 individual nights of Cen X-3 observations after application of the full set of the shower image parameter cuts, and for different brightnesses of the images.

\begin{tabular}{|c|c|c|c|c|c|c|c|c|c|c|c|c|}
\hline \multirow[b]{2}{*}{ Date } & \multicolumn{4}{|c|}{ brightness $>800$ digital counts } & \multicolumn{4}{|c|}{ brightness $>1500$ digital counts } & \multicolumn{4}{|c|}{ brightness $>2000$ digital counts } \\
\hline & on & off & $\operatorname{sig}$ & $\mathrm{gr} / \mathrm{cr}$ & on & off & $\operatorname{sig}$ & $\mathrm{gr} / \mathrm{cr}$ & on & off & $\operatorname{sig}$ & $\mathrm{gr} / \mathrm{cr}$ \\
\hline $01 / 03 / 97$ & 446 & 401 & 1.546 & 0.112 & 265 & 218 & 2.139 & 0.216 & 199 & 173 & 1.348 & 0.150 \\
\hline $03 / 03 / 97$ & 572 & 515 & 1.729 & 0.111 & 391 & 329 & 2.311 & 0.188 & 339 & 282 & 2.287 & 0.202 \\
\hline $04 / 03 / 97$ & 383 & 319 & 2.416 & 0.201 & 233 & 187 & 2.245 & 0.246 & 184 & 149 & 1.918 & 0.235 \\
\hline $01 / 06 / 97$ & 265 & 256 & 0.394 & 0.035 & 184 & 171 & 0.690 & 0.076 & 171 & 156 & 0.830 & 0.096 \\
\hline 02/06/97 & 455 & 383 & 2.487 & 0.188 & 304 & 262 & 1.765 & 0.160 & 260 & 219 & 1.873 & 0.187 \\
\hline $04 / 06 / 97$ & 200 & 185 & 0.764 & 0.081 & 146 & 143 & 0.176 & 0.021 & 131 & 138 & -0.427 & -0.051 \\
\hline $05 / 06 / 97$ & 323 & 295 & 1.126 & 0.095 & 218 & 203 & 0.731 & 0.074 & 182 & 169 & 0.694 & 0.077 \\
\hline $07 / 06 / 97$ & 394 & 377 & 0.612 & 0.045 & 273 & 258 & 0.651 & 0.058 & 231 & 215 & 0.758 & 0.074 \\
\hline 1997 Total & 3038 & 2731 & 4.042 & 0.112 & 2014 & 1771 & 3.950 & 0.137 & 1697 & 1501 & 3.466 & 0.131 \\
\hline $27 / 03 / 98$ & 689 & 628 & 1.681 & 0.097 & 411 & 378 & 1.175 & 0.087 & 296 & 280 & 0.667 & 0.057 \\
\hline $29 / 03 / 98$ & 661 & 589 & 2.036 & 0.122 & 424 & 347 & 2.773 & 0.222 & 309 & 243 & 2.809 & 0.272 \\
\hline $30 / 03 / 98$ & 364 & 373 & -0.332 & -0.024 & 226 & 220 & 0.284 & 0.027 & 174 & 163 & 0.599 & 0.067 \\
\hline $17 / 04 / 98$ & 182 & 178 & 0.211 & 0.022 & 109 & 98 & 0.765 & 0.112 & 78 & 78 & 0 & 0 \\
\hline $19 / 04 / 98$ & 339 & 336 & 0.115 & 0.009 & 232 & 216 & 0.756 & 0.074 & 183 & 167 & 0.855 & 0.096 \\
\hline $26 / 04 / 98$ & 59 & 56 & 0.280 & 0.054 & 45 & 39 & 0.655 & 0.154 & 39 & 32 & 0.831 & 0.219 \\
\hline $27 / 04 / 98$ & 473 & 441 & 1.058 & 0.073 & 371 & 334 & 1.394 & 0.111 & 313 & 270 & 1.781 & 0.159 \\
\hline $28 / 04 / 98$ & 272 & 293 & -0.883 & -0.072 & 206 & 220 & -0.678 & -0.064 & 172 & 181 & -0.479 & -0.050 \\
\hline $29 / 04 / 98$ & 151 & 126 & 1.502 & 0.198 & 113 & 96 & 1.176 & 0.177 & 87 & 85 & 0.152 & 0.024 \\
\hline 1998 Total & 3190 & 3020 & 2.157 & 0.056 & 2137 & 1948 & 2.957 & 0.097 & 1651 & 1499 & 2.708 & 0.101 \\
\hline $13 / 02 / 99$ & 431 & 451 & -0.673 & -0.044 & 323 & 337 & -0.545 & -0.042 & 263 & 266 & -0.130 & -0.011 \\
\hline $15 / 02 / 99$ & 78 & 66 & 1.00 & 0.182 & 42 & 30 & 1.414 & 0.400 & 30 & 16 & 2.064 & 0.875 \\
\hline $16 / 02 / 99$ & 923 & 902 & 0.492 & 0.023 & 614 & 602 & 0.344 & 0.020 & 492 & 495 & -0.095 & -0.006 \\
\hline $17 / 02 / 99$ & 922 & 889 & 0.775 & 0.037 & 643 & 611 & 0.904 & 0.052 & 536 & 479 & 1.789 & 0.119 \\
\hline $20 / 02 / 99$ & 206 & 212 & -0.293 & -0.028 & 164 & 170 & -0.328 & -0.035 & 144 & 146 & -0.117 & -0.014 \\
\hline $21 / 02 / 99$ & 355 & 294 & 2.39 & 0.207 & 248 & 210 & 1.778 & 0.181 & 210 & 172 & 1.944 & 0.221 \\
\hline 1999 Total & 2915 & 2814 & 1.334 & 0.036 & 2034 & 1960 & 1.171 & 0.0378 & 1675 & 1574 & 1.772 & 0.064 \\
\hline Grand Total & 9143 & 8565 & 4.344 & 0.067 & 6185 & 5679 & 4.646 & 0.089 & 5023 & 4574 & 4.583 & 0.098 \\
\hline
\end{tabular}

purpose the priority should be given to keeping in the data as many $\gamma$-ray events as possible, but trying at the same time to reasonably reduce the $\mathrm{CR}$ background events. In order to do so, we have applied in succession a set of significantly softer image parameter cuts, removing at the first step from the initial data only the events with the orientation parameter $\alpha \geq 45^{\circ}$ (to compare with the $\alpha \leq 30^{\circ} \mathrm{kept}$ in the fully-cut sets). This procedure reduces by a factor of $\sim 3$ the number of CR induced events, but does not significantly affect the $\gamma$-ray events. Analyses of these data did not reveal any statistically significant (i.e. corresponding to chance probability $p$ below e.g. $10^{-3}$ ) Rayleigh peak either around the first or the second harmonic of the X-ray pulsar.

The next set of data has been prepared applying a set of "soft" image parameter cuts: by confining the position of the image within the camera (parameter distance); and by discriminating the images using the width and the eccentricity parameters. This procedure can be rather efficient for suppressing CR background by a factor of $\sim 8-10$, although it may also remove some $\gamma$-ray events. Lastly, we have also searched for pulsar periodicity signs in the events after discriminating the images by their brightness, choosing only those with $>1500$ digital 
counts as compared to those with brightness $>800$ digital counts. This procedure effectively increases the energy threshold by a factor $\sim 2$, therefore it is efficient for the timing analysis only if the $\gamma$-ray spectrum is much harder than the differential spectrum of the local CRs with the power-law index $\alpha_{\mathrm{cr}} \simeq 2.7$. This idea stems from earlier theoretical predictions that the spectra of episodic $\gamma$-rays expected in the VHE domain from X-ray binaries may be anomalously hard due to very significant absorption on the thermal optical/UV photons produced either by the compact $\gamma$-ray source (Aharonian \& Atoyan 1991, 1996) or by the optical companion star in the particular case of Cen X-3 (Bednarek 2000).

The detailed analysis of all these data sets has revealed a strong Rayleigh power peak, corresponding to the chance probability for an artifact modulation of only $p \sim 6.8 \times 10^{-7}$ (see Fig. 2), in the data of observations on 21 Feb. 1999 at $P_{1}=2.3999 \mathrm{~s}$. This period is blue-shifted from the nominal second harmonic of the X-ray pulsar $\left(P_{0} / 2=2.408785 \mathrm{~s}\right.$, after correcting to the pulsar orbital phase $\phi \simeq 0.765$ for that data) by $6.6 \mathrm{msec}$, and implies a motion of the $\gamma$-ray source (if true) with respect to the neutron star with a velocity $>10^{3} \mathrm{~km} \mathrm{~s}^{-1}$. Interestingly enough, but not surprisingly, this peak is found in the data after both the soft image cuts and brightness $>1500$ digital counts criteria have been applied. For comparison, in Fig. 3 we show the results for the low energy threshold case, brightness $>800$ digital counts. The peaks disappear in the fully cut data, which is not surprising in light of the discussion above.

A simple estimate for an overall probability $p_{\text {tot }}$ to find a Rayleigh power $P_{\text {Ray }}$ such that $p_{0}=\exp \left(-P_{\text {Ray }}\right) \leq$ $6.8 \times 10^{-7}$ by chance can be derived taking into account that for a mean duration of our observations of Cen $\mathrm{X}-3$ per night is about $3 \mathrm{hr}$, there are on average about 18.6 IFF intervals in the search window 4.79-4.83s and twice that number in the second harmonic search window, 2.395-2.415 s. Because we have prepared $6=3 \times 2$ combinations of data (for different parameter cuts and brightnesses) for each of the 23 days, the overall number of trials is $N_{\mathrm{IFF}} \leq 8 \times 10^{3}$. This means that the overall probability of this peak arising by chance increases to $p_{\text {tot }} \simeq N_{\mathrm{IFF}} \times p_{0} \leq 5.4 \times 10^{-3}$.

Although the chance probability is still small it is not a conclusive argument for VHE $\gamma$-ray periodicity. In an attempt to gain better control of the hypothesis testing the analysis was repeated using a Bayesian technique (see Gregory \& Loredo 1992; Orford 2000 for details). This method applies Bayesian probability theory by comparing the phase distribution of a constant model for the signal to members of a class of models with periodic structure. The periodic models describe the signal plus background with a stepwise function, resembling a histogram, of $\mathrm{m}$ phase bins per period. In the case of a periodic model, the non-uniformity in phase is characterised by the varying contents of the phase bins. Although the number of phase bins needed to detect any light curve and the origin of phase are unknowns, these "nuisance" parameters can
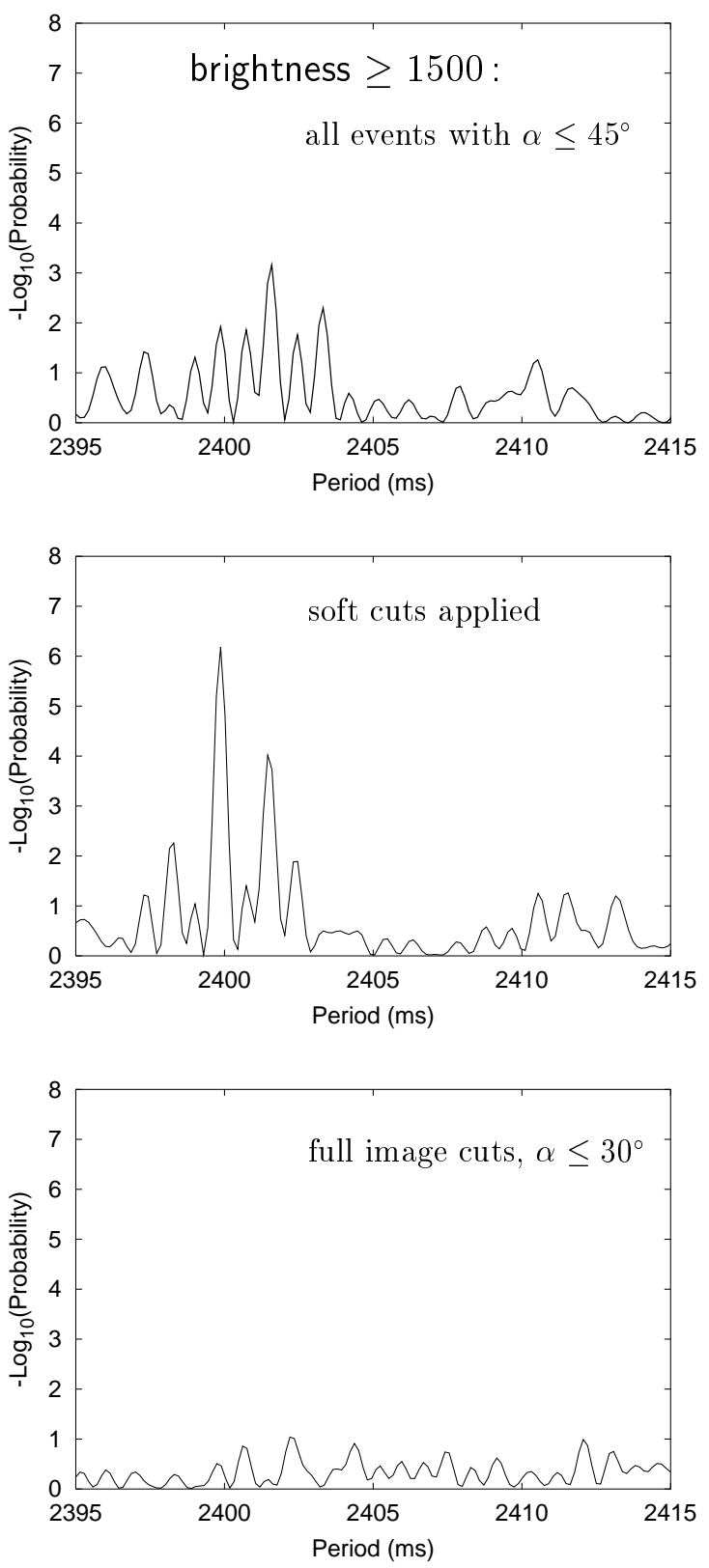

Fig. 2. Rayleigh power probabilities in the search window around the half-period (the second harmonic) $P_{0} / 2$ of the X-ray pulsar in the data sets of February 21, 1999 observations after application of image parameter cuts with different hardness.

be marginalised (integrated out); an important technical advantage of Bayesian inference over standard frequentist statistics. Since an arbitrary postulated light curve may be of any shape the method automatically applies Ockham's razor, in that models with fewer variables are automatically favoured unless the evidence from the data more than compensates for models of greater complexity. If there are $m$ phase bins the average rate $A=\frac{1}{m} \sum_{j=1}^{m} r_{j}$ and the fraction of the total rate in phase bin $j$ is $f_{j}=\frac{r_{j}}{m A}$. The likelihood function is shown to reduce to

$P\left(D \mid \omega, \phi, A, \boldsymbol{f}, M_{m}\right)=\Delta t^{N}(m A)^{N} \mathrm{e}^{-A T}\left(\prod_{j=1}^{m} f_{j}^{n_{j}}\right)$ 

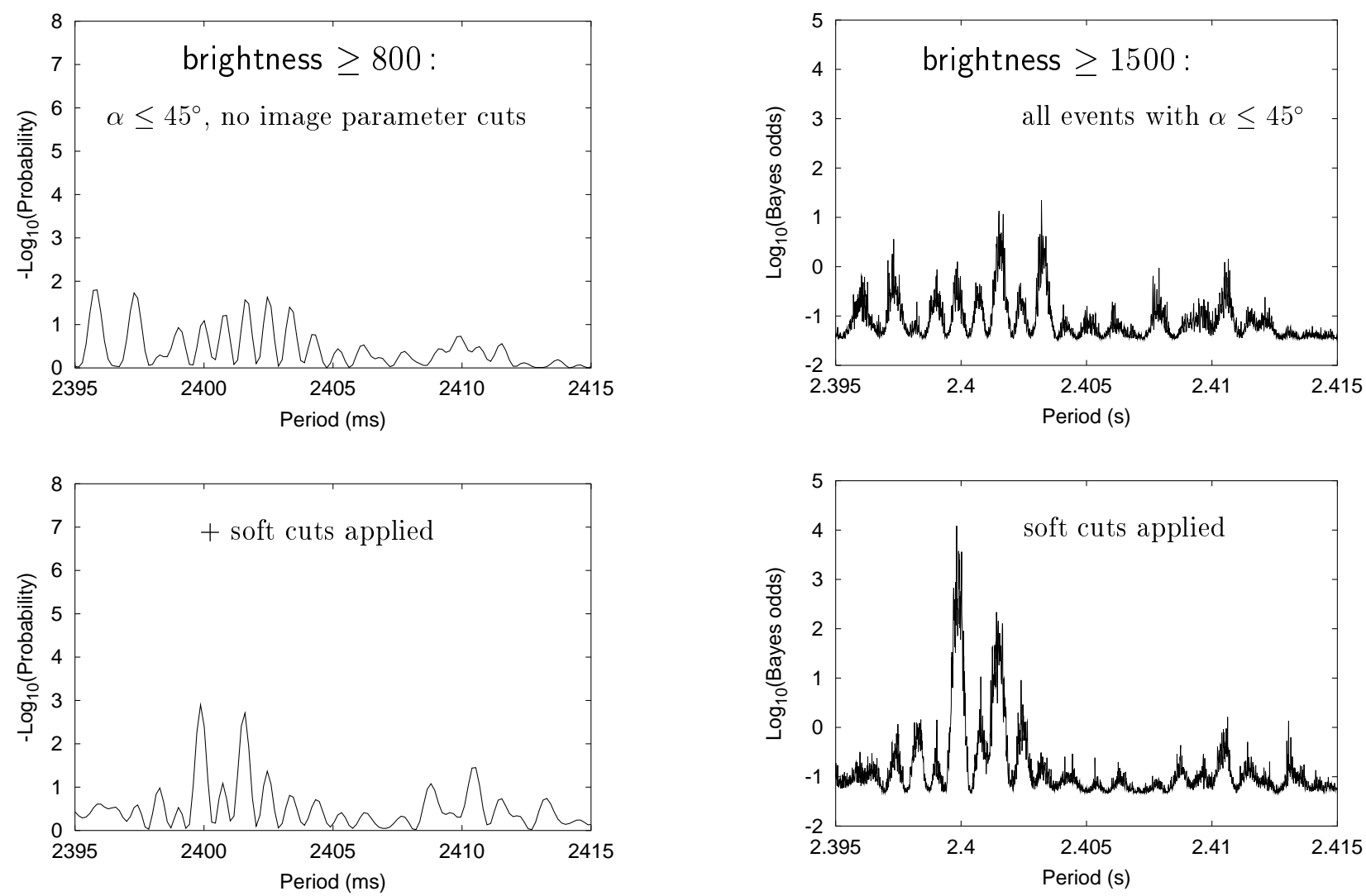

Fig. 3. Same as in Fig. 2, but for the events with parameter brightness $>800$ digital counts.

where $\omega$ is the postulated angular frequency, $\phi$ the starting phase, $\boldsymbol{f}$ the set of $m$ values of $f_{j}$ and $n_{j}$ being the number of events occurring in bin $j$.

The joint prior density for the parameters $\omega, \phi, A, \boldsymbol{f}$ is

$p\left(\omega, \phi, A, \boldsymbol{f} \mid M_{m}\right)=p\left(\omega \mid M_{m}\right) p\left(\phi \mid M_{m}\right) p\left(A \mid M_{m}\right) p\left(\mathbf{f} \mid M_{m}\right)$.

The prior densities are

- $p\left(\phi \mid M_{m}\right)=1 / 2 \pi$, this assumes any starting phase is equally likely;

- $p\left(A \mid M_{m}\right)=1 / A_{\max }$, this assumes that $A$ does not change during the observation and any value of $A$ from $A=0$ to $A=A_{\max }$ is possible;

$-p\left(\omega \mid M_{m}\right)=\frac{1}{\omega \ln \left(\omega_{\text {hi }} / \omega_{\text {low }}\right)}$, where $\left[\omega_{\mathrm{hi}}, \omega_{\text {low }}\right]$ is the least informative prior for the range of $\omega$;

- $p\left(\boldsymbol{f} \mid M_{m}\right)=(m-1) ! \delta\left(1-\sum_{j=1}^{m} f_{j}\right)$, where $\delta$ denotes the dirac $\delta$-function.

The assignment of the priors beforehand is all that is needed before comparing the likelihoods of the models. The two models are equally likely a priori and so the prior likelihood of the non-periodic model $\left(M_{1}\right)$ is $p\left(M_{1}\right)=1 / 2$ and that for a periodic model $\left(M_{m}, m=2, m_{\max }\right)$ is $p\left(M_{m} \mid I\right)=1 / 2 \nu$, where $\nu=m_{\max }-1$.

The final result for the odds $O$ in favour of a periodic model and against a uniform model of phase when the

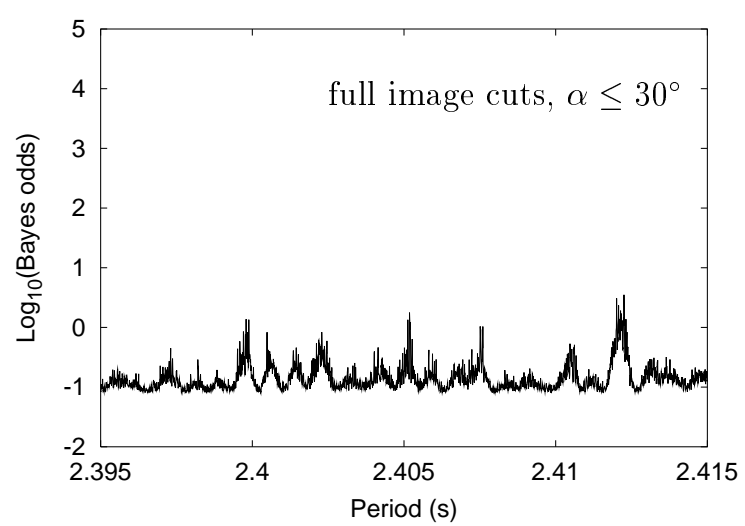

Fig. 4. Similar to Fig. 2, but for the Bayesian odds analyses.

phase and period are unknown (as in our case) is

$$
\begin{aligned}
O_{m 1}= & \frac{1}{2 \pi \ln \left(\frac{\omega_{\text {hi }}}{\omega_{\text {low }}}\right)} \frac{N !(m-1) !}{(N+m-1) !} \\
& \times \int_{\omega_{\text {low }}}^{\omega_{\text {hi }}} \frac{\mathrm{d} \omega}{\omega} \int_{0}^{2 \pi} \mathrm{d} \phi \frac{m^{N}}{W_{m}(\omega, \phi)}
\end{aligned}
$$

where $W_{m}(\omega, \phi)$ is the number of ways that the set of $n_{j}$ counts can be made by distributing $N$ counts in $m$ bins:

$W_{m}(\omega, \phi)=\frac{N !}{\prod_{j=1}^{m} n_{j} !}$ 
and $n_{j}$, the number of events placed in the $j$ th phase bin, depends on $\omega, \phi$ and $m$. The overall likelihood of periodicity is then found from

$O_{\text {per }}=\sum_{m=2}^{m_{\max }} O_{m 1}$.

The attractiveness of the Bayesian technique is that the overall probability of the hypothesis of a time-modulated signal does not scale linearly with the number of trials as in the Rayleigh test, but with the $\ln$ (period range searched). The periodicity search is therefore only penalised by the fact that the period is uncertain within a range and not by the number of times that region is searched. In Fig. 4 we show the Bayesian odds results for the same data sets as used in Fig. 2 for the Rayleigh statistics. Formally, the peak in the median panel in Fig. 4 means that the hypothesis that the data set does contain a signal modulated at the period $P_{1}=2.3998 \mathrm{~s}$ is $N_{\text {odds }}=1.2 \times 10^{4}$ times more probable than the hypothesis of a uniform time distribution. Note that the positions and the qualitative behaviour of the 2 highest peaks in Figs. 2 and 4 are similar.

Finally, it is worth noting the earlier reports in the 1980's about the detection of anomalously pulsating episodic VHE $\gamma$-ray emission from Her X-1 by three independent groups (Whipple, Lamb et al. 1988; Haleakala, Resvanis et al. 1988; and Los Alamos, Dingus et al. 1988), offset from the main period by $\sim 0.16 \%$. It is also worth noting that Her X-1 had a short episode $(\Delta t \leq 1 \mathrm{hr})$ of $\gamma$-ray emission reported as being detected simultaneously and independently by the University of Durham (Chadwick et al. 1987) and the Whipple Observatory (Gorham et al. 1986) groups, with both pulsation frequencies and estimated fluxes being consistent in the initial uncut data of both groups. After application of hard AZWIDTH parameter cuts which had earlier produced the best results for the Crab Nebula signal (Weekes et al. 1989), the pulsations in all Her X-1 data of the Whipple group disappeared (Reynolds et al. 1991). This, however, is just the outcome that one would expect, as we have discussed above in this section. Taking into account the rather steep spectrum of the Crab Nebula compared to the one expected from Her X-1 (Aharonian \& Atoyan 1996), we may here hypothesise that an application of much softer parameter cuts, that would first of all secure a larger number of possible $\gamma$-ray events in the remaining data, could possibly have a better chance to enhance the initial signal.

\section{Possible models}

\subsection{General considerations}

The results of observations of Cen X-3 in both HE and VHE $\gamma$-ray domains allow us to significantly constrain different models of $\gamma$-ray production in this X-ray binary. The first informative result is the absence of any signs of orbital modulation of the $\gamma$-ray signal in either of these energy domains. Because of the presence of a very luminous
O-type star close to the pulsar the VHE radiation produced at the pulsar orbit would be significantly absorbed on the optical/UV photons from the companion prior to escape from the system. Therefore the $\gamma$-ray source cannot be placed close to the neutron star. This conclusion is also in agreement with the fact of detection of $\gamma$-rays by EGRET (Vestrand et al. 1997), and probably also by the Mark 6 telescope, in the phase of complete eclipse of the X-ray pulsar.

Thus, at least for this particular binary any model assuming production of $\gamma$-rays directly by the pulsar, or models invoking the accretion disc around the neutron star as a site for $\gamma$-ray production (e.g. Chanmugam \& Brecher 1985; Katz \& Smith 1988; Raubenheimer \& Smit 1997), can be excluded from the list of reasonable options. At the same time, the $\gamma$-ray source must be genetically connected to the neutron star. This requirement follows not from the effect of $\gamma$-ray modulations with the pulsar spin, which would still need additional confirmation by future observations, but from the general considerations of the source energetics.

Indeed, the radiation flux detected by EGRET during 2 weeks in 1994 corresponds to an average source luminosity of $L_{\gamma}(100 \mathrm{MeV} \leq E \leq 10 \mathrm{GeV}) \simeq 5 \times 10^{36} \mathrm{erg} \mathrm{s}^{-1}$ (Vestrand et al. 1997), and the mean flux detected in 1997-1999 by the Mark 6 telescope corresponds to a luminosity $L_{\gamma}(>400 \mathrm{GeV}) \sim 10^{36} \mathrm{erg} \mathrm{s}^{-1}$. For the parent relativistic particles, either electrons or protons, these luminosities inevitably imply an acceleration power $P_{\text {acc }} \sim$ $10^{37} \mathrm{erg} \mathrm{s}^{-1}$ or higher. The optical/UV luminosity of the Krzeminski star corresponding to the bolometric magnitude $M_{\text {bol }} \simeq-9$ (Hutchings et al. 1979) is really high, $L_{\mathrm{bol}} \simeq 10^{39} \mathrm{erg} / \mathrm{s}^{2}$. It would suffice if a small fraction of this luminosity could somehow be converted to the acceleration of relativistic particles. In principle, this might be due to a radiatively driven supersonic stellar wind and subsequent production of shocks in such winds. Meanwhile, for the characteristic speed of the stellar wind $v \sim 10^{3} \mathrm{~km} \mathrm{~s}^{-1}$ and the mass-loss rate $\dot{M} \sim 10^{-6} M_{\odot} \mathrm{yr}^{-1}$ (Clark et al. 1988) the entire kinetic energy of the wind produced by the Krzeminski star makes only $P_{\text {wind }}=$ $\dot{M} v^{2} / 2 \simeq 3 \times 10^{35} \mathrm{erg} \mathrm{s}^{-1}$.

Any reasonable possibility for production of a kinetic power exceeding $10^{37} \mathrm{erg} \mathrm{s}^{-1}$ in Cen X-3/V779 Cen binary is therefore to be attributed to the neutron star. For the $4.8 \mathrm{~s}$ spin period of Cen X-3 the classical mechanism of the magnetic dipole radiation falls several orders of magnitude below the values required. Therefore the only remaining principal option for the prime energy source for the acceleration of relativistic particles in this system remains the kinetic energy of the inner accretion disc formed around the neutron star. Most of the kinetic energy of the disc ends up on the neutron star surface in the form of the thermal plasma energy responsible for the observed

\footnotetext{
${ }^{2}$ Note that for a massive star with $M \sim 20 M_{\odot}$ this is already comparable with, but still significantly below the Eddington luminosity $L_{\mathrm{Edd}}=1.3 \times 10^{38}\left(\mathrm{M} / \mathrm{M}_{\odot}\right) \mathrm{erg} \mathrm{s}^{-1}$.
} 
$\mathrm{X}$-rays with the luminosity reaching (in the high state) $L_{\mathrm{X}} \geq 10^{38} \mathrm{erg} \mathrm{s}^{-1}$. It is possible, however, that a significant fraction of the accretion disc energy can be ejected from the system producing powerful outflows in the form of two-sided jets observed at least in some class of close X-ray binaries currently identified as "microquasars" (see Mirabel \& Rodriguez 1999 for a review), which may contain either a stellar-mass black hole or a neutron star. A spectacular example of a microquasar containing an accreting neutron star that produces powerful subrelativistic $\left(v_{\text {jet }} \approx 0.26 c\right)$ jets is SS 433 , with the jet kinetic energy estimated in the range from $\sim 10^{39} \mathrm{erg} \mathrm{s}^{-1}$ to $4 \times 10^{40} \mathrm{erg} \mathrm{s}^{-1}$ (see Margon \& Anderson 1989; Panferov 1999). In this regard it is also worth noting the recent report by Jernigan et al. (2000) of the detection of high-frequency QPOs ("quasi-periodic oscillations") in the X-ray emission of Cen X-3, which is a characteristic feature of microquasars.

The principal scenarios for $\gamma$-ray production in Cen X3 are thus connected with powerful jets of plasma driven by the inner accretion disc. In one such scenario these jets would create strong shocks while propagating through the rather dense wind driven by the O-star. Subsequent acceleration of particles on these shocks could result in the creation of a $\gamma$-ray source around the region of jet propagation (or its damping) on large spatial scales $l_{\mathrm{s}}$ comparable with, or more probably significantly exceeding, the size of the binary $\sim 10^{12} \mathrm{~cm}$. Obviously, such a spatially extended model will not be able to explain episodes of pulsed $\gamma$-ray emission that may have been detected from the system. Evidence for pulsed emission from Cen X-3 is far from conclusive, but if it is there it probably constitutes only a fraction of the overall $\gamma$-radiation of Cen X-3. An interpretation of the "anomalously" pulsed $\gamma$-ray episodes may be possible in the framework of compact-source model scenarios that would suppose direct interaction of the jet either with material ejected from the inner parts of the accretion disc, or with a dense external target that might accidentally fall under the jet. Below we will discuss these model possibilities.

\subsection{Spatially extended source model}

In the framework of this model that assumes acceleration of particles on the shocks driven into the supersonic (and therefore favourable for generation of shocks) wind of the O-star by jets appearing from the inner accretion disc, the source size $l_{\mathrm{s}} \gg 10^{12} \mathrm{~cm}$, so that the $\gamma$-ray source is surrounding the X-ray pulsar. This circumstance allows us to exclude with a significant confidence the hadronic $\left(\pi^{0}-\right.$ decay) origin of $\gamma$-rays for the spatially extended model.

Indeed, the energy loss time of protons due to inelastic collisions with ambient gas with a density $n_{\text {gas }}$ (in terms of nucleons, or "H-atoms") is

$t_{\mathrm{pp}}=\left(K_{\mathrm{p}} \sigma_{\mathrm{pp}} n_{\mathrm{gas}} c\right)^{-1} \simeq 2.5 \times 10^{5}\left(\frac{n_{\mathrm{gas}}}{10^{10} \mathrm{~cm}^{-3}}\right)^{-1} \mathrm{~s}$

where $K_{\mathrm{p}} \approx 0.45$ is the inelasticity coefficient and $\sigma_{\mathrm{pp}} \sim$ $30 \mathrm{mb}$ is the cross-section of nuclear interactions of protons at $\mathrm{GeV}$ energies which slowly increases with the particle energy. Taking then into account also that in pp-collisions only $\simeq 1 / 3$ of the initial energy of relativistic particles is eventually transformed to $\gamma$-rays (the rest goes to the secondary electrons and neutrinos), the total energy $W_{\mathrm{p}}$ of relativistic protons which is needed for $\gamma$-ray production with luminosity $L_{\gamma}$ is estimated as

$W_{\mathrm{p}} \simeq 3 t_{\mathrm{pp}} \times L_{\gamma}$.

For a $\gamma$-ray source extending up to characteristic distances $R_{\mathrm{s}}$ measured from the centre of the O-star, the source volume can be generally written as $V_{\mathrm{s}}=f_{\mathrm{V}} \times 4 \pi R_{\mathrm{s}}^{3} / 3$ where $f_{\mathrm{V}} \leq 1$ is the volume filling factor. The energy density of relativistic protons $w_{\mathrm{p}}=W_{\mathrm{p}} / V_{\mathrm{s}}$ should then be compared with the energy density $w_{\mathrm{gr}}=\frac{G M m_{\mathrm{p}} n_{\mathrm{gas}}}{R_{\mathrm{s}}}$ of the thermal gas in the gravitational field of the massive companion star with $M \simeq 20 M_{\odot}$ which defines the gravitational confinement potential of the binary. Obviously, in any reasonable scenario the energy density of relativistic charged particles that can accumulate (be frozen) in the ambient plasma should not exceed the gravitational field energy density, otherwise the relativistic particle gas cannot be confined to a scale $R_{\mathrm{S}}$ but would act to inflate the source, also strongly affecting the gas dynamics in the binary system. Then from the condition $w_{\mathrm{p}} \leq w_{\text {gr }}$ we can estimate the maximum $\gamma$-ray luminosity $L_{\pi^{0}}^{\max }$ that can be produced by relativistic protons:

$$
\begin{aligned}
L_{\pi^{0}}^{\max } & =\frac{4 \pi}{9} f_{\mathrm{V}} G M m_{\mathrm{p}} K_{\mathrm{p}} \sigma_{\mathrm{pp}} N_{\mathrm{H}}^{2} c \\
& \simeq 2.5 \times 10^{34} f_{\mathrm{V}}\left(\frac{M}{20 M_{\odot}}\right)\left(\frac{N_{\mathrm{H}}}{10^{23} \mathrm{~cm}^{-2}}\right)^{2} \mathrm{erg} \mathrm{s}^{-1}
\end{aligned}
$$

Here $N_{\mathrm{H}}=n_{\text {gas }} \times R_{\mathrm{s}}$ is the gas column density of the $\gamma$-ray source which we have normalized to $10^{23} \mathrm{~cm}^{-2}$. This is a reasonable maximum value for $N_{\mathrm{H}}$ that corresponds to the gas density $n_{\text {gas }} \leq 10^{11} \mathrm{~cm}^{-3}$ of the wind at distances $R_{\mathrm{s}} \geq 10^{12} \mathrm{~cm}$ (Clark et al. 1988). Equation (3) shows that the maximum luminosity to be expected in $\gamma$-rays of hadronic origin is orders of magnitude below the $\gamma$-ray luminosities detected from Cen X-3.

It should be noted that Eq. (3) is actually a rather universal upper limit for the $\pi^{0}$-decay $\gamma$-ray luminosity that can be produced (quasi-stationarily) by a source gravitationally confined to a central mass $M$. In particular, this relation is valid for $\pi^{0}$-decay $\gamma$-rays that can be produced in the accretion discs of neutron stars or black holes (including supermassive $\mathrm{BH}$ in AGNs). For Cen X-3 we should substitute $M \rightarrow M_{\mathrm{n}} \simeq 1.2 M_{\odot}$ and take into account that for any reasonable disc geometry $f_{\mathrm{V}}$ cannot exceed $\sim 0.1$. Then even assuming $N_{\mathrm{H}} \simeq 8 \times 10^{23} \mathrm{~cm}^{-2}$ which is the characteristic maximum column density accumulated across the accretion disc of Cen X-3, as deduced by Nagase et al. (1992) from the X-ray line variations in the pre-eclipse dips, we conclude that any hadronic model invoking the accretion disc as a site for $\gamma$-ray production would be far too inefficient for an explanation of the observed fluxes, or otherwise the relativistic proton gas would blow up the accretion disc itself. 
Considering now models that assume a leptonic origin of $\gamma$-rays, it becomes crucial for the existence of a quasi-stationary large-scale source that the energy loss time of the electrons in Cen X-3 is much shorter than $t_{\mathrm{pp}}$. At distances $R \geq 1.5 R_{\mathrm{O}}$ from the O-star the energy density of the thermal optical/UV radiation field is $w_{\mathrm{UV}}=L_{\mathrm{bol}} / 4 \pi R^{2} c$, therefore the cooling time of an electron with Lorentz-factor $\gamma$ due to inverse Compton (IC) radiation can be estimated as

$t_{\mathrm{IC}} \simeq 1.1 \times 10^{4}\left(R / 10^{12} \mathrm{~cm}\right)^{2} \gamma^{-1} \mathrm{~s}$.

This estimate is valid in the Thomson limit for IC scattering when the parameter $b=4 \gamma\left(\epsilon_{0} / m_{\mathrm{e}} c^{2}\right)<1$. For the O-star in Cen X-3 with $T \sim 35000 \mathrm{~K}$ the mean energy of the thermal photons is $\epsilon_{0} \sim 3 k T \simeq 9 \mathrm{eV}$, therefore the transition from the Thomson limit to the relativistic Klein-Nishina limit of IC scattering occurs at $\gamma \sim \gamma_{\mathrm{KN}}=1.4 \times 10^{4}$. Thus, for electrons with $\gamma \sim 10^{3}-10^{4}$ responsible for production of high-energy $\gamma$-rays the IC cooling time is orders of magnitude smaller than the cooling time of relativistic protons ${ }^{3}$ given by Eq. (1). For electrons with $\gamma \geq \gamma_{\mathrm{KN}}$ the IC loss time does not drop further as Eq. (4) would predict, but rather increases, so that for VHE electrons the IC cooling time at distances $R$ is about $\left(10^{2}-10^{3}\right) \times\left(R / 10^{12} \mathrm{~cm}\right)^{2}$. Thus, for these electrons as well the case is still $t_{\mathrm{IC}} \ll t_{\mathrm{pp}}$, which leads to a total energy $W_{\mathrm{e}}$ needed in relativistic electrons which is much smaller than $W_{\mathrm{p}}$.

Thus, a spatially extended $\gamma$-ray source assumes diffusive shock acceleration (e.g. see Drury 1983; Blandford $\&$ Eichler 1987) of the electrons on spatial scales $R_{\mathrm{S}}$ comparable or exceeding the characteristic length scale of the binary. In order to estimate a plausible size $R_{\mathrm{s}}$, let us estimate the characteristic time needed for acceleration up to energies $E \sim 10 \mathrm{TeV}$. Using the results of Lagage \& Cesarski (1983), in the Bohm diffusion limit this time can be expressed as

$t_{\mathrm{acc}} \simeq 7 \times 10^{4} \frac{E}{10 \mathrm{TeV}}\left(\frac{B}{1 \mathrm{G}}\right)^{-1}\left(\frac{v_{\mathrm{sh}}}{3000 \mathrm{~km} \mathrm{~s}^{-1}}\right)^{-2} \mathrm{~s}$,

where $v_{\text {sh }}$ is a mean shock speed. Since the characteristic speed of the stellar wind in Cen X-3 is $v_{\mathrm{w}} \sim 10^{3} \mathrm{~km} \mathrm{~s}^{-1}$, the normalization of $v_{\mathrm{sh}}$ to $\sim 3000 \mathrm{~km} \mathrm{~s}^{-1}$ used above seems reasonable. Now taking into account that because of advection in the plasma wind all relativistic particles leave the radial scales $R$ at least on the convective escape timescale $t_{\mathrm{esc}} \simeq R / v_{\mathrm{w}}$, the characteristic size of the region required in this scenario for production of multi-TeV electrons should satisfy the condition $t_{\mathrm{acc}}(10 \mathrm{TeV}) \leq t_{\mathrm{esc}}$. As it follows from our calculations below, the magnetic fields of order $B \sim 1 \mathrm{G}$ in the stellar wind of Cen X-3 would not contradict the fact of non-detection of radio fluxes from Cen X-3 (see Vestrand et al. 1997). Therefore, from the condition $t_{\mathrm{esc}} \geq t_{\mathrm{acc}}$, the size of the $\mathrm{TeV} \gamma$-ray source is estimated as $R_{\mathrm{s}} \geq 5 \times 10^{12} \mathrm{~cm}$. On the other hand, the

\footnotetext{
${ }^{3}$ Note that with increasing $R$ the gas density $n_{\text {gas }}$ drops approximately as $\propto R^{-2}$, so $t_{\mathrm{pp}} \propto R^{2}$ similar to $t_{\mathrm{IC}}$.
}

source size should not significantly exceed $10^{14} \mathrm{~cm}$ because of the fast decline of the density of the UV photon field from the O-star, which essentially reduces the efficiency of the IC $\gamma$-ray production (as $t_{\mathrm{IC}}>t_{\mathrm{esc}}$ ).

Note that $R_{\mathrm{S}}$ is much larger than the radius $R_{\mathrm{O}}=$ $8.6 \times 10^{11} \mathrm{~cm}$ of the Krzeminski star or the distance $a=1.3 \times 10^{12} \mathrm{~cm}$ between the stars. This implies a source volume filling factor $f_{\mathrm{V}} \sim 1$. More importantly, the large $R_{\mathrm{S}}$ suggests that the $\gamma$-ray source is quasi-stationary on time scales $\geq 1$ day.

Provided that the volume occupied by the shock fronts (i.e. where the particle acceleration occurs) is significantly smaller than the $\gamma$-ray source entire volume, one can describe the energy distribution of relativistic electrons $N(E)$ by a kinetic equation (see e.g. Ginzburg \& Syrovatskii 1964):

$$
\frac{\partial N}{\partial t}=\frac{\partial}{\partial E}(P N)-\frac{N}{t_{\mathrm{esc}}}+Q
$$

where $Q \equiv Q(E, t)$ is the rate of the electron acceleration/injection, and $P=-\mathrm{d} E / \mathrm{d} t$ corresponds to overall electron energy losses averaged over the volume of the source. The injection spectrum of the electrons is approximated as

$$
Q(E) \propto E^{-\alpha_{\mathrm{e}}} \exp \left(-E / E_{0}\right),
$$

with $\alpha_{\mathrm{e}} \sim 2$ typical for the diffusive acceleration on the strong shock fronts, and $E_{0} \geq 10 \mathrm{TeV}$ for the exponential cutoff energy.

In Fig. 5 we show the spectra of non-thermal radiation from radio wavelengths up to the HE $\gamma$-ray band calculated for such a quasi-stationary spatially extended source with $R_{\mathrm{S}}=6 \times 10^{12} \mathrm{~s}$, assuming injection of relativistic electrons with an overall power $P_{\text {acc }}=2 \times 10^{37} \mathrm{erg}$. In order to assess a contribution to the overall fluxes due to electrons taken away to large distances with the stellar wind, the calculations are done in a two-zone model approach (see Atoyan et al. 2000 for details), which assumes that there is no acceleration of fresh electrons at $R>R_{\mathrm{s}}$ (zone 2), but there is rather an injection of electrons escaping from the main source (zone 1) at the rate $Q^{\prime}=N(E) / t_{\text {esc }}$. In Fig. 5 the fluxes of synchrotron, bremsstrahlung and IC radiation produced in zone 2 are shown by thin lines. Obviously these fluxes are negligible compared with the respective ones produced in the region $R \leq R_{\mathrm{S}}$ where the effective electron acceleration is supposed. It tells us that for most of the electrons the characteristic cooling time inside the source is indeed significantly shorter than their escape time. This is apparent also in Fig. 6 where the energy distribution of relativistic electrons in zone 2 is shown by a dot-dashed line. Note an unusual profoundly concave shape of the electron distribution which is due to the Klein-Nishina effect in the IC energy losses of VHE electrons in the thermal radiation field of the O-star.

The full dot in Fig. 5 corresponds to the upper flux limit $S_{\nu} \leq 70 \mathrm{mJy}$ at $5 \mathrm{GHz}$ in the direction of Cen X-3 (Vestrand et al. 1997). The heavy solid curve in this figure 


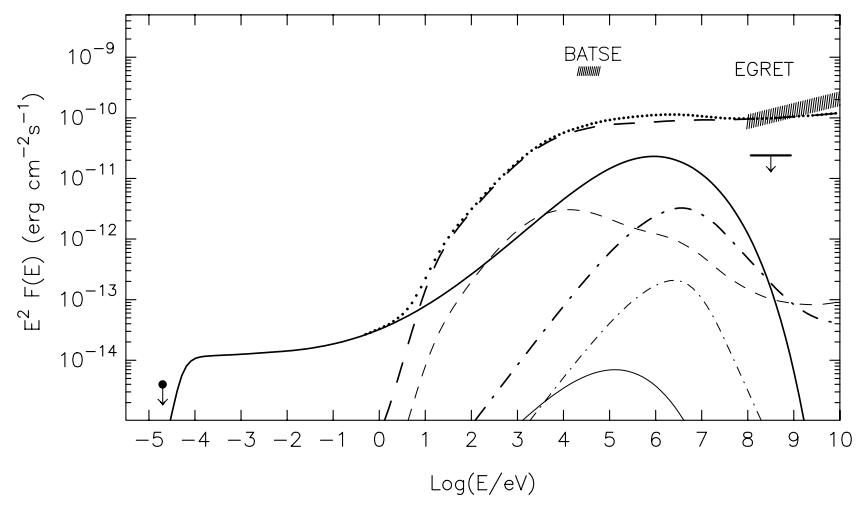

Fig. 5. The spectra of synchrotron (solid lines), bremsstrahlung (dot-dashed lines), and inverse Compton (dashed lines) radiations expected when calculated in the framework of a quasi-stationary model with $R_{\mathrm{s}}=6 \times 10^{12} \mathrm{~s}$, assuming a magnetic field $B=0.7 \mathrm{G}$, and injection spectrum of electrons with $\alpha_{\mathrm{e}}=2.0, E_{0}=15 \mathrm{TeV}$, and $P_{\mathrm{acc}}=2 \times 10^{37} \mathrm{erg}$. Radiation fluxes produced outside the source (zone 2, see text) are shown by thin lines.

shows that due to synchrotron self-absorption at frequencies below $10-15 \mathrm{GHz}$ the values of the magnetic field up to $\sim 1 \mathrm{G}$ at distances $\leq 10^{13} \mathrm{~cm}$ can still be accepted. However an assumption of a stronger magnetic field would result in significant synchrotron fluxes at sub-mm wavelengths which would then already be observed.

In Fig. 5 we also show a typical level of X-ray fluxes detected by BATSE from Cen X-3 in the active ("high") state. These fluxes are predominantly of thermal origin, and much higher than the non-thermal fluxes. Note however that the latter could, in principle, show up in the hard X-ray/soft $\gamma$-ray band, $E \sim(0.1-10) \mathrm{MeV}$, where the thermal radiation of the neutron star drops. In the region of HE $\gamma$-rays the IC radiation flux $\alpha \simeq 2$ is somewhat steeper than the October 1994 flux of Cen X-3 with a hard mean differential power-law index $\alpha_{\text {obs }}=1.81$ detected by EGRET (hatched region in Fig. 5). However, if we take into account the reported uncertainty in the power-law index $\pm \Delta \alpha=0.37$, the model appears in good agreement with the EGRET data (see Fig. 7, where the upper boundary of the hatched zone at $0.1-10 \mathrm{GeV}$ energies shows the median flux, while the lower one corresponds to $\alpha_{\mathrm{obs}}=2.18$ ).

In Fig. 7 IC $\gamma$-ray fluxes extending beyond $10 \mathrm{TeV}$ are presented. The solid curve corresponds to the unabsorbed flux produced in the source. The principal target photons for the IC scattering of the electrons in this model is the thermal UV radiation of the O-star, the contributions due to X-ray (3-dot-dashed) and synchrotron (dot-dashed) photons being negligible at all $E$. The heavy dashed line shows the fluxes of $\gamma$-rays escaping the source, after taking into account their absorption in the UV photon field. Calculations assume that $\gamma$-rays are produced uniformly at the scales $R \leq R_{\mathrm{s}}$.

In order to demonstrate a possible impact of photoabsorption, in Fig. 7 we show by full dots the flux that would

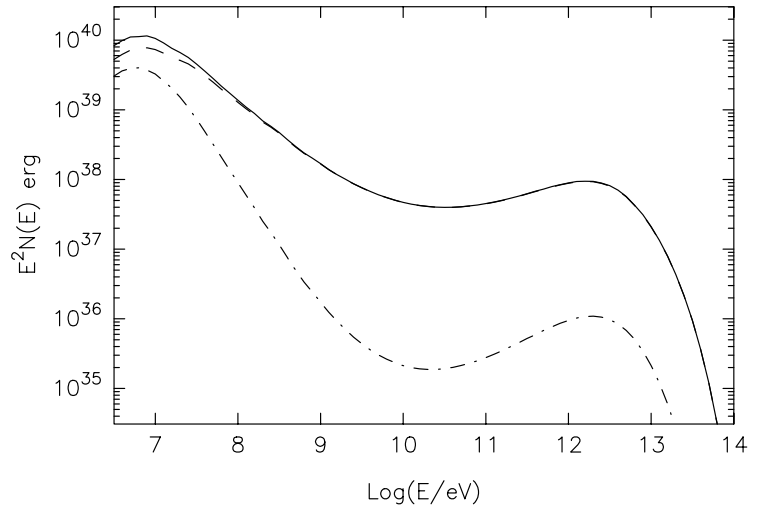

Fig. 6. The energy spectra of relativistic electrons formed in zones 1 (dashed) and zone 2 (dot-dashed), and the total spectrum (solid line) for the extended source model with parameters in Fig. 5

escape the binary if the same unabsorbed $\gamma$-radiation were to be produced close to the neutron star in the orbital phase $\phi \sim \pm 0.25$. In that case the optical depth $\tau_{\gamma \gamma}(E)$ would reach a maximum $\simeq 7$ at energies $E \sim 200 \mathrm{GeV}$, and $\gamma \gamma$-absorption would remain very significant in the entire region from $E \geq 30 \mathrm{GeV}$. Because $\tau_{\gamma \gamma}$ depends strongly on $\phi$, essentially becoming less than 1 at a phase of $\phi \sim 0.5$, a strong orbital modulation of VHE $\gamma$-ray fluxes produced close to the neutron star would be expected. In our calculations we did not take into account a possible contribution connected with the development of pair-photon cascades in the atmosphere of Cen X-3, which in practice remains small and cannot in any way significantly compensate the impact of absorption on the spectra of VHE $\gamma$-rays (see Bednarek 2000).

The total amount of energy accumulated in relativistic electrons shown in Fig. 6 is $W_{\mathrm{e}}=2.4 \times 10^{40} \mathrm{erg}$. For comparison of the efficiencies of hadronic and leptonic models, in Fig. 7 we show by stars the fluxes of $\pi^{0}$-decay $\gamma$-rays produced by relativistic protons with the same amount of total energy, $W_{\mathrm{p}}=2.4 \times 10^{40} \mathrm{erg}$.

It should nevertheless be said that the energy $W_{\mathrm{e}}$ is still significantly larger than the total potential energy $W_{\mathrm{G}}$ of the plasma in the gravitational field of the binary at scales $\leq 10^{13} \mathrm{~cm}$. The latter can be expressed directly through the mass loss rate $\dot{M}=4 \pi m_{\mathrm{p}} n_{\text {gas }} R^{2} v_{\mathrm{w}}$ of the Ostar if we approximate the stellar wind speed as a constant, $v_{\mathrm{w}}(R) \simeq 10^{3} \mathrm{~km} \mathrm{~s}^{-1}$ :

$W_{\mathrm{G}} \simeq 2 \times 10^{39} \dot{M}_{-6} \frac{M_{\mathrm{O}}}{20 M_{\odot}}\left(\frac{v_{\mathrm{w}}}{10^{3} \mathrm{~km} \mathrm{~s}^{-1}}\right)^{-1} \mathrm{erg}$,

where $\dot{M}_{-6} \equiv \dot{M} / 10^{-6} M_{\odot} \mathrm{yr}^{-1}$. However, $W_{\mathrm{e}}$ is comparable with the total kinetic energy of the wind

$W_{\text {kin }} \simeq 3.2 \times 10^{40} \dot{M}_{-6}\left(\frac{v_{\mathrm{w}}}{10^{3} \mathrm{~km} \mathrm{~s}^{-1}}\right)\left(\frac{R}{10^{13} \mathrm{~cm}} \mathrm{erg}\right)$.

It then implies that this model can still be magnetohydrodynamically self-consistent because the pressure of the relativistic electron gas can be "balanced" by the kinetic 


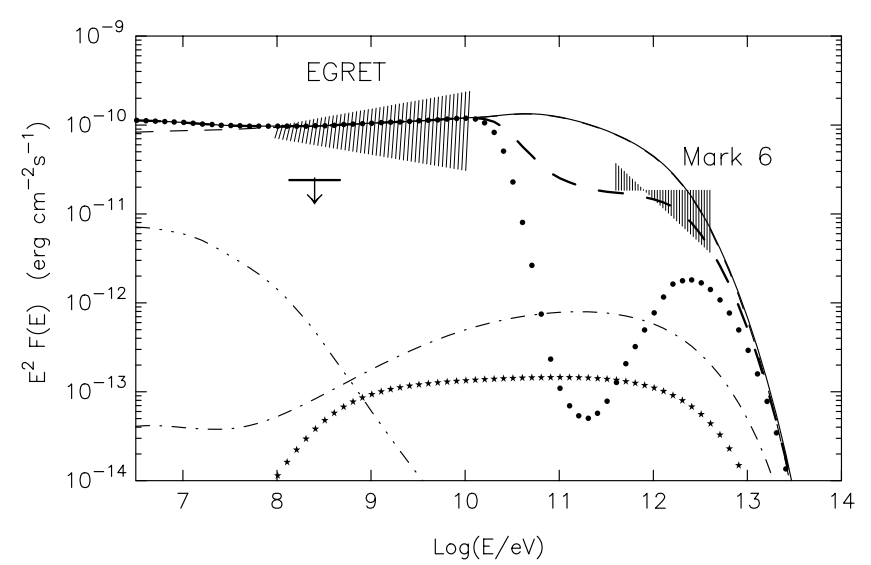

Fig. 7. The spectra of IC $\gamma$-rays calculated for the spatially extended source with parameters in Fig. 5. Contributions to the total unabsorbed IC radiation (solid line) due to upscattering of the UV radiation of the companion O-star (thin dashed line; distinguished only at $E<10^{8} \mathrm{eV}$ ), X-ray pulsar photons (3-dot-dashed line) and synchrotron photons (dot-dashed line) are shown. The heavy dashed line shows the spectrum escaping the source. The heavy dotted line shows the fluxes that would be expected if the same unabsorbed radiation were produced at the pulsar orbit; the stars show the unabsorbed radiation of hadronic origin (see text). The hatched region at $\mathrm{TeV}$ energies corresponds to the average flux detected by the Mark 6 telescope during 1997 (which is higher than the mean for 19971999, but closer to 1994) calculated for differential power-law indices between $\alpha_{\gamma}=2$ and 3. The range of differential fluxes, from median to low, detected by EGRET during October 1994, as well as the upper flux limit for earlier observation period, are also shown.

( ram) pressure. Obviously, the electrons in these circumstances should contribute very significantly to the gas dynamics of the stellar wind.

It is worth comparing here requirements that would follow from similar arguments in the case of a spatially extended hadronic model. In order to explain the observations one would require an overall energy in relativistic protons almost 3 orders of magnitude larger than the flux of gamma-rays of hadronic origin (shown by stars in Fig. 7 ); i.e. $W_{\mathrm{p}}$ would exceed $10^{43} \mathrm{erg}$. Such a huge amount of energy at a scale of $R_{\mathrm{s}}=\leq 10^{13} \mathrm{~cm}$, as assumed for Fig. 7 , is very problematic to expect from a binary star generally, and is far beyond the gravitational confinement abilities of the star, as discussed above. Moreover, Eq. (9) predicts that one would then expect such a relativistic proton gas would drive a wind in Cen. X-3 moving at subrelativistic speeds $\left(v_{w}\right)$. Assuming a larger source size would not help, as might be hinted by the same Eq. (9), on the contrary it will make the situation even worse because of a fast drop off of the mean gas density at larger distances resulting in a fast increase of the overall energy of relativistic protons needed. Only a rather compact source that could provide gas densities much higher than are found in the O-star wind, so resulting in a reasonable $W_{\mathrm{p}}$, giving a reasonable chance to provide the observed fluxes (see below). Note, however, that this does not mean that in the framework of a large-scale model protons are not allowed to be accelerated with an efficiency comparable with the electrons.

The relativistic electron gas pressure in the source can be reduced further if we take into account that most of the energy in Fig. 6 is due to particles with $E \leq 100 \mathrm{MeV}$ which do not contribute to production of HE $\gamma$-rays. Then we can reduce $W_{\mathrm{e}}$ assuming a very hard spectrum of accelerated electrons with $\alpha_{\mathrm{e}}<2$. Note that taking into account non-linear modification effects at the strong shock fronts, power-law spectral indices of the shock accelerated particles even as hard as $\alpha_{\mathrm{e}}=1.5$ could be expected (Malkov 1997).

In Fig. 8 we show the $\gamma$-ray spectra calculated for $\alpha_{\mathrm{e}}=1.75$, and assuming an injection rate of electrons with $P_{\text {acc }}=6 \times 10^{36} \mathrm{erg} \mathrm{s}^{-1}$. In this case the total energy of the electrons in the region $R \leq R_{\mathrm{s}}=10^{13} \mathrm{~cm}$ is only $W_{\mathrm{e}}=4.1 \times 10^{39} \mathrm{erg}$. Note that in the HE $\gamma$-ray domain the shape of the model spectrum now agrees well with the median spectral index of the fluxes detected by EGRET in October 1994. Meanwhile, the absolute fluxes of HE $\gamma$-radiation are now in agreement with the upper flux limits reported for the 9 month earlier observing session of Cen X-3 by EGRET (Vestrand et al. 1997). For calculations in Fig. 8 we have chosen rather a small acceleration rate of electrons, $P_{\text {acc }}=6 \times 10^{36} \mathrm{erg} \mathrm{s}^{-1}$, in order to demonstrate that in principle in the framework of this quasi-stationary model with $\alpha_{\mathrm{e}}<2$ it is possible to expect episodes (on time scales $\geq 1 \mathrm{~d}$ ) of $\gamma$-radiation that could have been detected in the VHE $\gamma$-rays, but would be missed by EGRET.

In conclusion, a spatially extended quasi-stationary source model seems capable of providing a phenomenologically self-consistent explanation for most of the currently existing data of $\gamma$-ray observations of Cen X-3. The single observational feature which cannot be addressed by this model is an interpretation of possible modulations of the $\gamma$-ray emission with the pulsar spin period $P_{0}$. If genuine, such modulations would either require an alternative or an additional much more compact $\gamma$-ray source.

\subsection{Compact source models}

An obvious requirement on any model that would be able to explain a $\gamma$-ray emission modulated at the pulsar spin period $P_{0}$ is that the effective source radius $R_{\mathrm{s}}$ has to be noticeably smaller than $c P_{0} / 2$. For Cen X-3 therefore $R_{\mathrm{S}}$ should be $\leq 5 \times 10^{10} \mathrm{~cm}$. Another requirement is that the energy loss time $t_{\text {loss }}$ of relativistic particles producing pulsed $\gamma$-rays should also be smaller than $P_{0}$. Then for $t_{\text {loss }} \sim 1 \mathrm{~s}$ the energy density of relativistic particles can be estimated as $w_{\text {rel }} \geq 10^{4} \mathrm{erg} / \mathrm{cm}^{3}$ using the relation $W_{\text {rel }} \geq L_{\gamma} t_{\text {loss }}$ for the total particle energy in the source. Such high energy densities can be reached in the binary only in the inner accretion disc around the neutron star, which however cannot be a site for the $\gamma$-ray emission because of the absence of any indications for the 


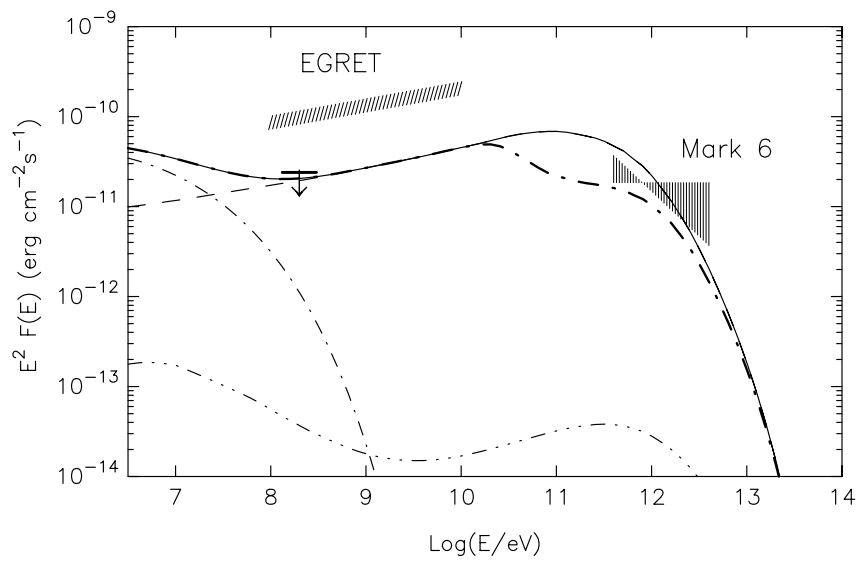

Fig. 8. Gamma-ray fluxes (heavy dot-dashed line) expected in the framework of a quasi-stationary source with $R_{\mathrm{s}}=10^{13} \mathrm{~cm}$, $B=1 \mathrm{G}$ in the case of a hard power-law spectrum of accelerated electrons with $\alpha_{\mathrm{e}}=1.75$, and total electron acceleration power $P_{\text {acc }}=6 \times 10^{36} \mathrm{erg} \mathrm{s}^{-1}$. Contributions due to IC (dashed line), synchrotron (thin dot-dashed line) and bremsstrahlung (3-dot-dashed line) radiation mechanisms are shown.

orbital modulation of the fluxes in both the HE and VHE domains, as discussed in Sect. 2 above. This implies that the source of pulsed $\gamma$-radiation in Cen X-3 cannot be confined, so that generally one would expect only rather short episodes of pulsed $\gamma$-ray emission.

As we have discussed above, a source of the observed energetic gamma-radiation in this X-ray binary should be connected with jets generated by the accretion disc around the neutron star. There are then two principal types of model, leptonic and hadronic, for the production of episodic pulsed radiation, both of which would assume a compact source ("clouds", "blobs") propagating in the jet. Pulsations could be produced if a powerful relativistic energy outflow, in the form of either electromagnetic Poynting flux or a beam of relativistic particles, modulated with the pulsar spin period propagates in the jet region and accelerates/injects relativistic particles in the source.

A hadronic "beam-target" model for production of pulsed VHE $\gamma$-radiation in X-ray binaries has been suggested earlier by Aharonian \& Atoyan $(1991,1996)$. This model assumes that a powerful beam of relativistic protons accelerated in the vicinity of the pulsar hits a dense plasma cloud that may appear in the jet propagation region. As it follows from Eq. (1), the gas density in the cloud should be very high, $n_{\text {gas }} \geq 10^{15} \mathrm{~cm}^{-3}$, in order to provide fast energy losses for the protons, $t_{\mathrm{pp}}<P_{0}=4.8 \mathrm{~s}$. For a cloud with a radius $R_{\mathrm{cl}} \sim 10^{10} \mathrm{~cm}$ this implies a cloud mass $M_{\mathrm{cl}} \geq 10^{22} \mathrm{~g}$. One may phenomenologically suppose that either such dense plasma blobs are ejected from the massive optical companion star, and then they may cross a conical region of jet propagation. We may also speculate that compact dense plasma blobs can be ejected from the accretion disc or be created in the jet region, and then the "beam-target" interaction takes place in the episodes when relativistic protons are sporadically accel- erated in the central engine (e.g. by the dynamo action, magnetic field line reconnection, etc.) forming relativistic proton beams streaming in the jets. this regard that very dense and compact $\left(\sim 10^{8} \mathrm{~cm}\right)$ gas clouds responsible for the observed optical emission in the powerful relativistic jets of the prominent binary SS 433, are supposed to be produced/condensed due to thermal instabilities in those jets (see Brinkmann et al. 1988; Panferov 1999).

A relativistic proton beam interacting with the gas in the cloud would produce $\pi^{0}$-decay $\gamma$-rays. Non-thermal radiation in this model will be contributed also by secondary electrons $\left(\mathrm{e}^{ \pm}\right)$. At the same time some fraction (a few per cent, see Aharonian \& Atoyan 1991) of the injected proton energy would inevitably go to Coulomb ("ionization") losses heating the cloud up to temperatures $T_{\mathrm{cl}} \sim(5-10) \times 10^{4} \mathrm{~K}$. This results in a very high density UV radiation field in the cloud, so that its opacity with respect to VHE $\gamma$-rays can be very high. The spectra of escaping $\gamma$-rays are then essentially defined by the pairphoton cascade developing in the cloud. At the same time, because of the high pressure created, the source would expand with a speed presumably of order of the sound speed, $\sim \sqrt{k T_{\mathrm{cl}} / m_{\mathrm{p}}}$, if not confined by some external pressure. Therefore the radiation spectra predicted in this expanding source should be rapidly evolving.

In Fig. 9 we show the non-thermal radiation spectra expected in this model at 4 different times $t$, from 5 min to 1 day (=86400 s), after a cloud with a mass $M_{\mathrm{cl}}=10^{22} \mathrm{~g}$ and an initial size $R_{\mathrm{cl}}=5 \times 10^{9} \mathrm{~cm}$ is hit by a powerful beam of relativistic protons at the distance $D_{\mathrm{cl}}=8 \times$ $10^{11} \mathrm{~cm}$ from the neutron star. The method of calculations used and other details of the model are found in Aharonian \& Atoyan (1996). The spatial angular density of the beam power needed in this model is rather high, $\mathrm{d} P_{\text {beam }} / \mathrm{d} \Omega=$ $3 \times 10^{41} \mathrm{erg} \mathrm{s}^{-1} \mathrm{sr}^{-1}$ in Fig. 9. This implies that in order to have reasonable total energetics, the relativistic proton beam should be collimated to within a few degrees. Thus, for the collimation angle $5^{\circ}$ the total beam power would be $P_{\text {beam }}=1.8 \times 10^{39} \mathrm{erg} \mathrm{s}^{-1}$. We remind the reader in this regard that the collimation angle of SS 433 jets is $\theta_{\mathrm{j}} \leq 1.4^{\circ}$, and the jet kinetic energy is estimated up to several times $10^{40} \mathrm{erg} \mathrm{s}^{-1}$ (e.g. Margon \& Anderson 1989).

Under the pressure of such a powerful beam the expanding clouds would be expelled from the binary reaching speeds of order $10^{4} \mathrm{~km} \mathrm{~s}^{-1}$. This is seen in Fig. 9 where we show the increasing distance $D_{\mathrm{cl}}$ of the cloud from the neutron star. The characteristic proton cooling times and the cloud's radius are also shown. For the cloud mass supposed in this case one could expect a pulsed emission only during the first $\leq 1 \mathrm{~h}$, since at $t=3 \mathrm{~h}$ the gas density in the cloud drops below $10^{15} \mathrm{~cm}^{-3}$ so that $t_{\mathrm{pp}}>P_{0}$. Note, however, that calculations in Fig. 9 assume an external pressure free expansion of the cloud with the sound speed.

In fact the expansion of a fast cloud may be significantly confined due to the ram pressure of the external medium $p_{\mathrm{ram}}=m_{\mathrm{p}} n_{\mathrm{gas}} v_{\mathrm{cl}}^{2}$. For a gas density in the wind of the O-star declining as $R^{-2}$, the maximum distance scale $\left(R_{\text {conf }}\right)$ in the binary up to which the ram pressure will 
be able to confine the cloud's expansion can be estimated from the condition $p_{\text {ram }} \geq p_{\text {rel }}$, where $p_{\text {rel }}=w_{\text {rel }} / 3$ is the pressure of relativistic particles. For $w_{\text {rel }} \geq 10^{4} \mathrm{erg} \mathrm{s}^{-1}$ estimated above this results in

$R_{\mathrm{conf}} \leq 5 \times 10^{12} \dot{M}_{-6}^{1 / 2}\left(\frac{v_{\mathrm{cl}}}{10^{4} \mathrm{~km} \mathrm{~s}^{-1}}\right) \mathrm{cm}$,

where we have assumed $v_{\mathrm{w}}=10^{3} \mathrm{kms}^{-1}$ for the wind speed. Thus, for a mass loss rate $\dot{M} \leq 3 \times 10^{-6} M_{\odot} \mathrm{yr}^{-1}$ the source confinement time

$t_{\mathrm{conf}}=\frac{R_{\mathrm{conf}}}{v_{\mathrm{cl}}} \leq 5 \times 10^{3} \dot{M}_{-6}^{1 / 2} \mathrm{~s}$,

does not exceed a couple of hours, independently of the source speed. However, a high speed of source is important in order to provide $R_{\text {conf }} \gg 10^{12} \mathrm{~cm}$ because otherwise the flux of pulsed VHE $\gamma$-rays would be effectively suppressed. Eq. (10) then suggests that $v_{\mathrm{cl}} \sim 10^{4} \mathrm{~km} \mathrm{~s}^{-1}$ or larger. Note that both Eqs. (10) and (11) limitations relate not only to the hadronic model but in particular to the leptonic model for interpretation of pulsed $\gamma$-ray emission considered below in this section.

An interesting feature in Fig. 9 is a profound absorption of $\gamma$-radiation in the thermal UV radiation field of the cloud itself, and not only from that of the O-star. It leads to an unusually hard spectrum of VHE $\gamma$-rays predicted by this model at all initial stages $t \leq(1-2) \mathrm{h}$ when the pulsed signal is produced. In this regard, it is worth remembering that the most significant indications for a pulsed signal in our 21 February 1999 data are found in the air shower events corresponding to enhanced brightness.

Considering now a compact source with a leptonic origin for the pulsed $\gamma$-radiation, note that in this case we cannot assume that electrons are accelerated close to the pulsar and then supplied in a relativistic beam into the cloud because the life time of HE and VHE electrons in the radiation field of the companion star (Eq. (4)), is much shorter than their travel time to distances $\gg 10^{12} \mathrm{~cm}$ where the source of VHE $\gamma$-rays should be located, as discussed above.

The principal leptonic model for Cen X-3 then basically corresponds to the model developed earlier for the microquasars, i.e. galactic sources with relativistic jets like GRS $1915+105$. For a detailed description of the model we refer to Atoyan \& Aharonian (1999). Generally, this model assumes that the inner accretion disc of the compact object in the binary, in our case the neutron star (or a galactic BH candidate for GRS $1915+105$ ) sporadically ejects a pair of clouds in two opposite directions to each other. In the case of stellar-mass black holes the speed of the ejecta may reach typically $\geq 0.9 c$, whereas in the case of neutron star discs significantly smaller jet speeds are assumed (see Mirabel \& Rodriguez 1999). These clouds should then be energized from the central engine by the relativistic wind (e.g. Poynting flux) propagating in the jet region and modulated with the pulsar spin period. The latter condition would not be needed if the pulsed $\gamma$-rays should not be explained. Note that certain indications that the pairs of ejecta in GRS $1915+105$ are continuously fed with relativistic energy from the central source are found in the data of radio observations of this microquasar (see Atoyan \& Aharonian 1999). Relativistic shocks should then be formed at the contact interface between the ejecta and the wind, providing efficient acceleration of electrons. This could result in modulations of the $\gamma$-ray signal with the pulsar spin period (Doppler shifted), which would inevitably disappear at times $t \geq t_{\text {conf }}$ (Eq. (11)).

In Fig. 10 we show the broad-band spectra of synchrotron and IC radiation predicted by this model at 3 different times after ejection of the cloud(s) from the accretion disc: $t=100 \mathrm{~s}$ (solid curves), $t=1 \mathrm{~h}$ (dashed), and $t=1$ day (dot-dashed). For calculations in Fig. 10 we have formally assumed that the cloud expands with a mean speed $\simeq 10^{7} \mathrm{~cm} / \mathrm{s}$ so that the cloud radius at $t \sim 1 \mathrm{~h}$ would still be $<c P_{0} / 2$. The magnetic field behavior in the expanding cloud is approximated as $B(R) \propto R^{-1.5}$, with a normalisation to $B_{0}=50 \mathrm{G}$ at $R_{0}=10^{10} \mathrm{~cm}$. In such high magnetic fields, especially at the very initial stages when the cloud is compact, one has to take into account that synchrotron losses prevent acceleration of electrons effectively beyond the energy $E_{\max } \simeq 50 / \sqrt{B / 1 \mathrm{G}} \mathrm{TeV}$. This limitation partially explains an effective suppression of $\mathrm{TeV}$ radiation in Fig. 5 at $t=100 \mathrm{~s}$. It is more important, however, that the synchrotron self-absorption at the initial stages effectively removes from the source all submillimetre and radio photons, which become the principal targets for IC production of VHE radiation in such a compact source.

An important difference between the hadronic and leptonic compact sources is that the leptonic model does not need a high gas density in the source, and the mass load in the cloud may be very low. In that case the Coulomb losses of relativistic electrons are very low, so the cloud will not be heated to very high temperatures when the absorption of VHE $\gamma$-rays in the cloud becomes important. Therefore only the absorption in the UV radiation field of the O-star may affect the fluxes of VHE $\gamma$-rays which we do not take into account in Fig. 9). For the spectra at $t=100 \mathrm{~s}$ this absorption can be rather strong, depending on the orbital phase of the pulsar. Note that at that times the spectral modifications are dominated by the absorption of VHE $\gamma$-rays in the thermal UV radiation field of the cloud itself. At the times $t=1 \mathrm{~h}$, which is the basic timescale for production of the pulsed VHE radiation, the source moving with $v_{\mathrm{cl}} \geq 10^{4} \mathrm{~km} \mathrm{~s}^{-1}$ will be at distances far enough from the O-star that the absorption of VHE $\gamma$ rays is not dramatic. Therefore the model predicts rather fast evolution of the VHE radiation spectra at the stage of pulsations. Note that this prediction is also valid for the hadronic compact source model (see Fig. 9).

Unlike the hadronic model, however, the leptonic jet model predicts very significant fluxes at later stages of the flare evolution, $t \geq 1 \mathrm{~d}$, when the pulsations disappear. It is also worth noting that the shape of the spectra in the $\mathrm{HE} \gamma$-ray domain in this model are much harder, close to 

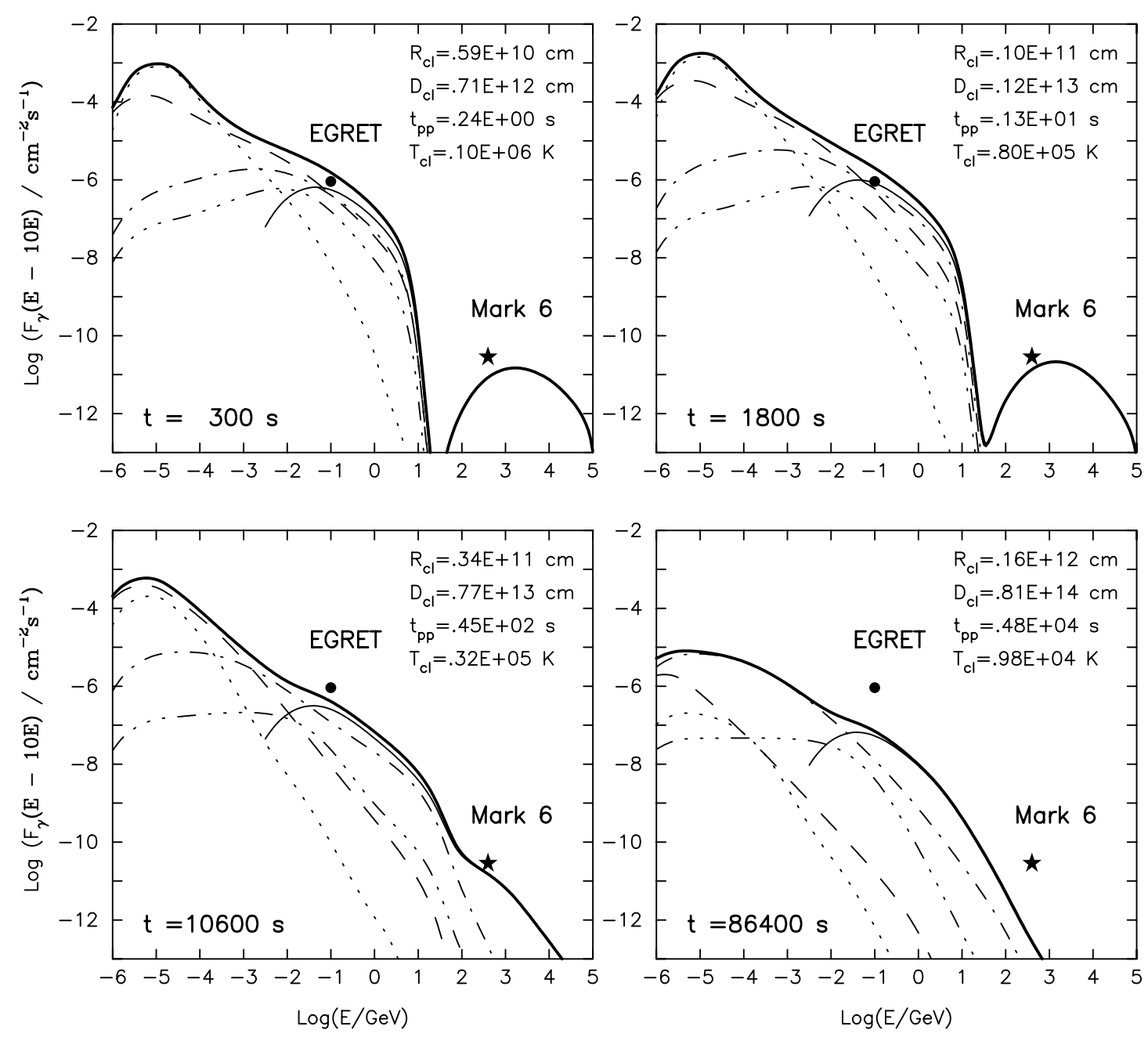

Fig. 9. The radiation spectra expected at different times from a compact heavy target with $M_{\mathrm{cl}}=10^{22} \mathrm{~g}$ falling under a powerful beam of relativistic protons. The fluxes and principal parameters of the cloud, as the radius $R_{\mathrm{cl}}$, the distance $D_{\mathrm{cl}}$ from the pulsar, the p-p interaction time $t_{\mathrm{pp}}$, and the cloud temperature $T_{\mathrm{cl}}$, are shown. The contributions of different radiation processes are shown by solid line ( $\pi^{0}$ decays), dashed line (synchrotron radiation), dot-dashed line (inverse Compton), 3-dot-dashed line (electron bremsstrahlung), dotted line ("Comptonization" - the multiple Compton scattered radiation in the thermal gas). The heavy solid line corresponds to the total spectrum escaping the cloud. Note that the integral fluxes here are integrated "per decade of the energy interval", which allows to better preserve the information about differential radiation flux as well.

the mean spectral flux detected by EGRET in October 1994 (the hatched region in Fig. 10). Besides that, this model can provide an answer to the question why the $\gamma$-ray signals seem to be more persistently detected (although at different times) by the Mark 6 telescope in the VHE domain than by EGRET at high energies (see dot-dashed curve in Fig. 10). In this regard note also that the heavy bar in Fig. 10 is the mean VHE $\gamma$-ray flux detected during 1997, which is higher by a factor of $\sim 2$ than the mean during 1997-1999.

Finally, in this section we also have to address the question of the characteristic frequencies of $\gamma$-ray pulsations that are to be expected. Because the location of the $\gamma$ ray source cannot coincide with the pulsar, one should not generally expect that the $\gamma$-ray pulsations would be coincident with the X-ray pulsations. In the framework of both models considered in this section we have to expect a difference between those pulsations because of the so called "double Doppler effect", which is the frequency shift $\Delta \nu=\nu-\nu_{0}$ due to re-emission/reflection of a pulsed signal from a target moving with a speed $\boldsymbol{u}$ (see Aharonian \& Atoyan 1991):

$\frac{\Delta \nu}{\nu_{0}}=\frac{u}{c} \frac{\cos (\hat{\boldsymbol{k} u})-\cos (\hat{\boldsymbol{p} u})}{1-(u / c) \cos (\hat{\boldsymbol{k}} \boldsymbol{u})}$

where $u \equiv|\boldsymbol{u}|$ is the cloud's velocity relative to the pulsar; $\hat{\boldsymbol{k}} \boldsymbol{u}$ and $\hat{\boldsymbol{p} u}$ are the angles that the vector $\boldsymbol{u}$ makes respectively with the $\gamma$-ray $(\boldsymbol{k})$ and the relativistic energy outflow $(\boldsymbol{p})$. Then for a compact source moving at high speed $v_{\mathrm{cl}} \sim 10^{4} \mathrm{~km} \mathrm{~s}^{-1}$ or more favoured by both models above, an interpretation of the shift of the Rayleigh power peak at $2.400 \mathrm{~s}$ from the nominal half-period (second harmonic) of the X-ray pulsar $P_{0} / 2=2.410 \mathrm{~s}$ would imply that both angles $\hat{\boldsymbol{k}} \boldsymbol{u}$ and $\hat{\boldsymbol{p} \boldsymbol{u}}$ were rather close, within $\leq 10^{\circ}$, to $90^{\circ}$. In principle, for Cen X-3 where the orbital plane of the 


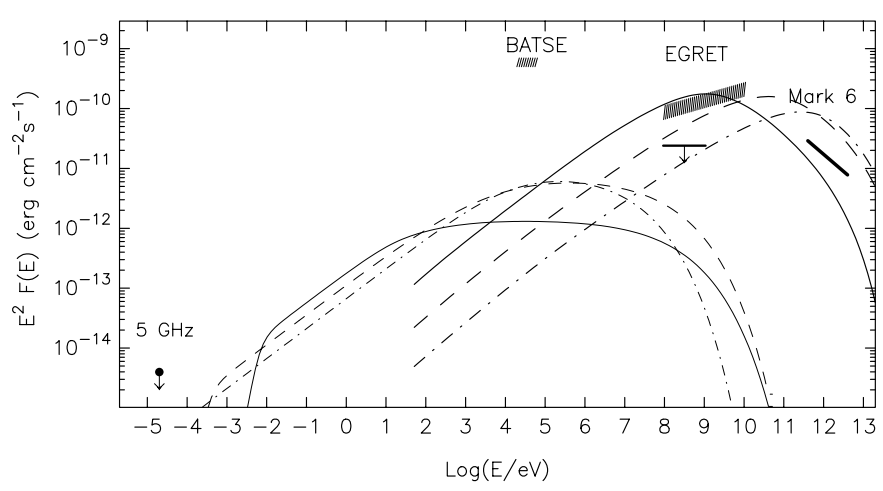

Fig. 10. The spectra of synchrotron (thin lines) and IC (heavy lines) radiations from a compact fast cloud ("ejecta") propagating in the jet(s) of Cen X-3 calculated for 3 different times after ejection: $t=100 \mathrm{~s}$ (solid lines), $t=1 \mathrm{~h}$ (dashed lines), and $t=1 \mathrm{~d}$ (dot-dashed lines). The total injection power in shockaccelerated electrons $P_{\text {acc }}=10^{37} \mathrm{erg} \mathrm{s}^{-1}$ is assumed. The heavy bar at $\mathrm{TeV}$ energies corresponds to the mean integral flux of $\mathrm{TeV} \gamma$-rays detected with the Mark 6 telescope in 1997 (see Chadwick et al. 2000) assuming a power-law differential spectrum with $\alpha=2.6$.

pulsar is close to the plane of sky this may still be possible if the jet is produced almost perpendicular to the orbital plane. However an interpretation of the $\gamma$-ray pulsations precisely at the X-ray pulsar frequency as reported by Vestrand et al. (1997) appears rather problematic. If confirmed by future observations, these pulsations could be reasonably explained only by a model assuming production of HE radiation very close to the pulsar which should then address again the problems of the energetics and confinement of such a source.

\section{Conclusions}

The analysis of the data of observations of Cen X-3 accumulated during 23 days of observations of Cen X-3 with the University of Durham Mark 6 imaging telescope during 1997-1999 confirms the presence of a $\gamma$-ray signal at the overall significance level $\geq 4.5 \sigma$ in agreement with our previous results (Chadwick et al. 2000). The behaviour of the signal, which does not drop but rather increases for images with enhanced brightness (see Table 1), indicates that the spectra of VHE $\gamma$-rays from Cen X-3 are probably much harder than the spectra of ambient cosmic rays with $\alpha_{\mathrm{CR}} \approx 2.7$.

No indications for any correlation of the VHE signal with the orbital motion of the pulsar, including the pulsar eclipse phase, is found. Given that a similar result is found by EGRET for HE $\gamma$-rays detected from Cen X-3, this effect excludes any close vicinity of the pulsar as a possible site for $\gamma$-ray production.

The analysis of the shower arrival times does not reveal any statistically significant peak of Rayleigh powers in most of the data, except for 1 observation day, the 21 Feb. 1999, showing a strong peak with an estimated probability of occurence of such a peak by chance, after taking into account the large number of IFF trials, of significantly below $10^{-2}$. The analysis using Bayesian statistics results in an even lower final probability, $p_{\text {Bay }} \simeq 7.5 \times 10^{-4}$.

It is indicative that the peak powers in both statistics are found in the data after application of soft image cuts, whereas they completely disappear when the full (hard) image cuts maximizing significance in the overall data of observations are applied. This behaviour is just what one should expect assuming that the modulated signal in the data of 21 Feb. 1999 is really connected with VHE $\gamma$-rays. The pulsed signal significantly increases with the threshold energy of the events, which indicates that the spectrum of pulsed events is hard as well.

The position of modulations found in 21 February 1999 data is blueshifted from the nominal half period of the $\mathrm{X}$-ray pulsations by $\delta \nu / \nu \simeq 3.7 \times 10^{-3}$. Such shifts should be reasonably expected in any model where the $\gamma$-rays are produced far away from the pulsar. Moreover, on the basis of the theoretical modelling in this paper, which suggests high speeds of compact source(s) needed for production of pulsed VHE $\gamma$-ray emission, we would generally expect that $\gamma$-ray pulsations could show even larger shifts, up to $|\Delta \nu| / \nu_{0} \sim 0.3$ in the case of ejecta moving with the speed $\leq 0.3 c$ such as in SS 433 .

The principal models for $\gamma$-ray production in Cen X-3 should be able to produce VHE radiation at distances significantly beyond the orbit of the pulsar, effectively at $R \geq 3 \times 10^{12} \mathrm{~cm}$. Meanwhile, high energies needed for the $\gamma$-ray fluxes in this binary can be provided only by the gravitational potential of the neutron star. Confirmation of the $\gamma$-ray fluxes from Cen X-3 by future observations would therefore confirm production of powerful jets by the inner accretion disc of this X-ray binary.

Except for the phenomenon of $\gamma$-ray pulsations (which obviously needs confirmation by forthcoming $\gamma$-ray detectors), an acceptable interpretation of the average fluxes currently reported in $\mathrm{HE}$ and $\mathrm{VHE} \gamma$-ray regions can in principle be provided in the framework of both spatially extended and compact source models, which may also "co-exist" operating together. The principal difference between these 2 model approaches is that the extended $\gamma$-ray source implies quasi-stationary emission on times scale of at least several days, and probably even weeks, whereas both hadronic and leptonic compact source models predict fast evolution of the $\gamma$-ray fluxes on time scales of a few hours, and with the "disappearance" of the source in a few days. Another informative feature could be the behaviour of the spectral fluxes at both high and very high energies.

An important prediction of both compact source models is that pulsed $\gamma$-ray emission can only be episodic, with a typical duration of no more than a few hours. For $\gamma$-rays of $E \sim 100 \mathrm{GeV}$ which are to be produced at large distances this is practically a model-independent requirement. However, $\gamma$-rays with $E \leq 10 \mathrm{GeV}$ can escape from the binary, even being produced relatively (but not very!) close to the X-ray pulsar. At these energies, therefore, 
detection of pulsed $\gamma$-rays in the eclipse phase of the X-ray pulsar would be very informative.

We can expect that future observations of Cen X-3 and other X-ray binaries with forthcoming sensitive $\gamma$-ray detectors in the $\geq 50 \mathrm{MeV}$ (GLAST) and $\geq 50 \mathrm{GeV}$ (HESS, CANGAROO-III, VERITAS) domains will provide large amounts of key information about high energy processes in these powerful galactic objects.

Acknowledgements. We are grateful to the UK Particle Physics and Astronomy Research Council for support of the project. AMA appreciates the hospitality of the Durham University during his visit to UK, and of the McGill University where his contribution to this paper could have been done. The work of AMA has been supported by the Royal Society visitor grant. This paper uses quick look results provided by the BATSE team.

\section{References}

Aharonian, F. A., \& Atoyan, A. M. 1991, ApJ, 381, 220

Aharonian, F. A., \& Atoyan, A. M. 1996, Space Sci. Rev., 75, 357

Armstrong, P., Chadwick, P. M., Cottle, P. J., et al. 1999, Experimental Astron., 9, 51

Ash, T. D. C., Reynolds, A. P., Roche, P., et al. 1999, MNRAS, 307,357

Atoyan, A. M., \& Aharonian, F. A. 1999, MNRAS, 302, 253

Atoyan, A. M., Tuffs, R. J., Aharonian, F. A., \& Völk, H. J. 2000, A\&A, 354, 915

Bednarek, W. 2000, A\&A, 362, 646

Blandford, R. D., \& Eichler, D. 1987, Phys. Rep., 154, 1

Brazier, K. T. S., et al. 1990, Proc. 21st Int. Cosmic Ray Conf. (Adelaide), 2, 296

Brazier, K. T. S., Carraminana, A., Chadwick, P. M., et al. 1990, ApJ, 350, 745

Brinkmann, W., Fink, H. H., Massaglia, S., Bodo, G., \& Ferrari, A. 1988, A\&A, 196, 313

Burderi, L., Di Salvo, T., Robba, N. R., La Barbera, A., \& Guainazzi, M. 2000, ApJ, 530, 429

Chanmugam, G., \& Brecher, K. 1985, Nature, 313, 767

Chadwick, P. M., Dipper, N. A., Kirkman, I. W., McComb, T. J. L., \& Orford, K. J. 1987, in Proc. NATO Workshop on Very High Energy Gamma-Ray Astronomy, ed. K. E. Turver (Dordrecht, Reidel), 121

Chadwick, P. M., Dickinson, M. R., Dipper, N. A., et al. 1998, ApJ, 503, 391

Chadwick, P. M., Lyons, K., McComb, T. J. L., et al. 1999, ApJ, 513, 161

Chadwick, P. M., Daniel, M. K., Lyons, K., et al. 2000, A\&A, 364,165

Clark, G. W., Minato, J. R., \& Mi, G. 1988, ApJ, 324, 974
Chodil, G., Mark, H., Rodrigues, R., et al. 1967, Phys. Rev. Lett., 19, 681

Dingus, B. L., Alexandras, D. E., Aller, R. C., Burman, R. L., \& Butterfield, K. B. 1988, Phys. Rev. Lett., 61, 1906

Dowthwaite, J. C., Harrison, A. B., Kirkman, I. W., et al. 1984, Nature, 309, 691

Drury, L. O'C. 1983, Rep. Prog. Phys., 46, 973

Giacconi, R., Gursky, H., Kellog, E., Schrier, E., \& Tananbaum, H. 1971, ApJ, 167, L67

Ginzburg, V. L., \& Syrovatskii, S. I. 1964, Origin of Cosmic Rays (Pergamon Press, London)

Gorham, P. W., Cawley, M. F., Fegan, D. J., et al. 1986, ApJ, 309, 114

Gregory, P. C., \& Loredo, T. J. 1992, ApJ, 398, 146

Hutchings, J. B., Cowley, A. P., Crampton, D., van Paradijs, J., \& White, N. E. 1979, ApJ, 229, 1079

Jernigan, J. G., Klein, R. I., \& Arons, J. 2000, ApJ, 530, 875

Joss, P. S., \& Rappaport, S. A. 1984, ARA\&A, 22, 537

Katz, J. I., \& Smith, I. A. 1988, ApJ, 326, 733

Kelley, R. L., Rappaport, S., Clark, G. W., \& Petro, L. D. 1983, ApJ, 268, 790

Krzeminski, W. 1974, ApJ, 192, L135

Lagage, P. O., \& Cesarsky, C. J. 1983, A\&A, 118, 223

Lamb, F. K., Pethick, C. J., \& Pines, D. 1973, ApJ, 184, 271

Lamb, R. C., Cawley, M. F., Fegan, D. J., et al. 1988, ApJ, 328, L13

Malkov, M. A. 1997, ApJ, 485, 638

Margon, B., \& Anderson, S. F. 1989, ApJ, 347, 448

Mirabel, I. F., \& Rodriguez, L. F. 1999, ARA\&A, 37, 409

Nagase, F. 1989, PASJ, 41, 1

Nagase, F., Corbet, R. H. D., Day, C. S. R., et al. 1992, ApJ, 396, 147

North, A. R., et al. 1990, Proc. 21st Int. Cosmic Ray Conf. (Adelaide), 2, 275

Orford, K. J. 2000, J. Phys. G: Nucl. Part. Phys., R1

Panferov, A. A. 1999, A\&A, 351, 156

Pringle, J. E., \& Rees, M. J. 1972, A\&A, 21, 1

Raubenheimer, B. C., \& Smit, H. J. 1997, Astropart. Phys. 7., 63

Resvanis, L. K., Szentgyorgyi, A., Hudson, J., et al. 1988, ApJ, 328, L9

Reynolds, P. T., Cawley, M. F., Fegan, D. J., et al. 1991, ApJ, 382,640

Schreier, E. J., Levinson, R., Gursky, H., et al. 1972, ApJ, 172, L79

Tuohy, I. R. 1976, MNRAS, 174, 45

Vestrand, W. T., \& Eichler, D. 1982, ApJ, 261, 251

Vestrand, W. T., Sreekumar, P., \& Mori, M. 1997, ApJ, 483, L49

Weekes, T. C., Cawley, M. F., Fegan, D. J., et al. 1989, ApJ, 342,379

Weekes, T. 1992, Space Sci. Rev., 59, 314

White, N. E., Swank, J. H., \& Holt, S. S. 1983, ApJ, 270, 711 